\title{
Allatostatin Immunoreactivity in the Honeybee Brain
}

\author{
Sabine Kreissl, ${ }^{*}$ Christine Strasser, and C. Giovanni Galizia \\ Department of Biology, University of Konstanz, D-78457 Konstanz, Germany
}

\begin{abstract}
Information transmission and processing in the brain is achieved through a small family of chemical neurotransmitters and neuromodulators and a very large family of neuropeptides. In order to understand neural networks in the brain it will be necessary, therefore, to understand the connectivity, morphology, and distribution of peptidergic neurons, and to elucidate their function in the brain. In this study we characterize the distribution of substances related to Dip-allatostatin I in the honeybee brain, which belongs to the allatostatin-A (AST) peptide family sharing the conserved c-terminal sequence -YXFGL-NH ${ }_{2}$. We found about 500 AST-immunoreactive (ASTir) neurons in the brain, scattered in 18 groups that varied in their precise location across individuals. Almost all areas of the brain were innervated by ASTir fibers. Most ASTir neurites formed networks
\end{abstract}

within functionally distinct areas, e.g., the antennal lobes, the mushroom bodies, or the optic lobes, indicating local functions of the peptide. A small number of very large neurons had widespread arborizations and neurites were found in the corpora cardiaca and in the cervical connectives, suggesting that AST also has global functions. We double-stained AST and GABA and found that a subset of ASTir neurons were GABA-immunoreactive (GABAir). Double staining AST with backfills of olfactory receptor neurons or mass fills of neurons in the antennal lobes and in the mushroom bodies allowed a more fine-grained description of ASTir networks. Together, this first comprehensive description of AST in the bee brain suggests a diverse functional role of AST, including local and global computational tasks.

INDEXING TERMS: neuropeptides; olfactory system; brain structure; colocalization; GABA; Apis mellifera

Neurons communicate chemically using neurotransmitters that are released presynaptically and which elicit a postsynaptic effect via transmitter selective receptors. There are three types of neurotransmitters, with the distinction among them becoming increasingly fuzzy: "classical" neurotransmitters such as glutamate, acetylcholine, and $\gamma$-aminobutyric acid (GABA) are released via clear vesicles, and generally act point-to-point at a localized synapse. Neuromodulators (e.g., biogenic amines such as dopamine, serotonin) are released by dense vesicles or via transmembrane transporters (Katz, 1999; Falkenburger et al., 2001), and generally act on large brain areas. The third group is the largest and consists of the neuropeptides. These are released by dense vesicles, in some instances at the synapses in a point-to-point fashion, akin to the classical neurotransmitters and often together with another transmitter; in other instances peptides are released extrasynaptically with a less local effect, akin to neuromodulators (Katz, 1999; Nässel and Homberg, 2006; Salio et al., 2006). The family of known neuropeptides already forms the largest family of interneuronal messengers, and it is likely that many remain to be discovered. Furthermore, each neuropeptide is likely to be associated with its own cognate G-protein-coupled receptor (Claeys et al., 2005). Thus, peptides and their cognate receptors form a toolbox that gives the brain the capacity to create a large variety of neurons with distinct physiological properties. Conversely, neuroscientists can use the diversity to unravel the morphology and physiology of chemically defined neural populations.

In this article we study the morphology of neurons expressing peptides belonging to the allatostatin-A (AST) group in the honeybee brain. Insects in general, and honeybees in particular, are very good model systems for studying the neurochemical basis of behavior. On the one side, their brains are relatively small $\left(\approx 10^{6}\right.$ neurons as

Additional supporting information may be found in the online version of this article.

Grant sponsor: DFG; Grant number: GA 524/8-1; Grant sponsor: German Bundesministerium für Bildung und Forschung; Grant number: $01 \mathrm{GQ077.}$

*CORRESPONDENCE TO: Dr. Sabine Kreissl, University of Konstanz, Department of Biology, Fach M 624, D-78457 Konstanz, Germany. E-mail: s.kreiss|@uni-konstanz.de 
compared to $10^{11}$ neurons in humans) and the arrangement of neuropils is relatively stereotypical, to a degree that some neurons can be identified individually. On the other side, their behavior is sufficiently complex, including learning and memory and close to cognitive capacities (Takeda, 1961; Galizia and Menzel, 2001; Menzel and Giurfa, 2001). Honeybees have a large complement of neuropeptides. Gene expression and immunohistochemical studies dealt with some of the better-known peptides including tachykinin and FMRF-like peptides (Schürmann and Erber, 1990; Eichmüller et al., 1991; Takeuchi et al., 2003, 2004) and GCCK (Strausfeld et al., 2000). After the complete sequencing of the bee's genome (Weinstock et al., 2006), coding sequences for some of the known receptors and arthropod neuropeptides were found by homology searches. MALDI-TOF analyses of the bee brain's peptidome also revealed the presence of many peptides, including members of the AST family (Weinstock et al., 2006; Audsley and Weaver, 2006; Hauser et al., 2006; Hummon et al., 2006; Predel and Neupert, 2007).

AST peptides share the conserved C-terminal sequence -YXFGL-NH ${ }_{2}$ (Bendena et al., 1999). They were first isolated from the cockroach Diploptera punctata, where they inhibit juvenile hormone synthesis in the corpora allata, hence their name (Stay and Tobe, 2007). They have been found in the nervous system of all insects analyzed so far, including cockroach (Belles et al., 1994), locusts (Veelaert et al., 1996), Lepidoptera (Duve et al., 1997; Utz and Schachtner, 2005; Utz et al., 2007), and Diptera (Duve et al., 1993; Yoon and Stay, 1995).
Five individual amidated ASTs have been predicted from an AST-A prohormone sequence deduced from an annotated genomic sequence. Three of these (Apime-AST 2, Apime-AST 3, Apime-AST 4, sensu Audsley and Weaver, 2006) share a serine (S), whereas Apime-AST 5 has a histidine $(H)$ as posttyrosyl residue. Apime-AST 1 displays an asparagine $(N)$ in the respective position and an isoleucine (I) instead of the common leucine (L) in the c-terminal position. lons with measured masses consistent with the three predicted sequences of Apime-AST 2, Apime-AST 3, and Apime-AST 4 were detected by mass spectrometry in extracts of the honeybee brain, with Apime-AST 4 being most abundant (Audsley and Weaver, 2006; Hummon et al., 2006).

AST exerted an inhibitory effect in all studies that examined its physiological effect in arthropods. In cockroaches, locusts, and blowflies AST inhibits spontaneous hindgut contractions or oviduct contractions (Duve and Thorpe, 1994; Duve et al., 1995; Veelaert et al., 1996). AST also inhibits peristaltic foregut activity in blowflies and locusts (Duve and Thorpe, 1994; Zilberstein et al., 2004). In crustaceans, AST decreases synaptic transmission by pre- and postsynaptic mechanisms at neuromuscular junctions (Kreissl et al., 1999) and inhibits the pyloric rhythm of the stomatogastric ganglion (Skiebe and Schneider, 1994; Dircksen et al., 1999) Furthermore, it increases spike-time precision within this network (Billimoria et al., 2006).

AST is often colocalized with other transmitters. In the locust central complex, AST-immunoreactive (ASTir) neurons also stain for serotonin and/or for GABA (Vitzthum

\begin{tabular}{|c|c|c|c|}
\hline & & ions & \\
\hline & & Lo & Lobula \\
\hline$A 32, A 65$ & Glomeruli 32 and 65 , respectively, innervated by $T 1$ & IPC & Lateral protocerebrum \\
\hline $\mathrm{A} 33, \mathrm{~A} 42$ & Glomeruli 33 and 42 innervated by $T 1$ & IPed & Lateral pedunculus \\
\hline AL & Antennal lobe & IPs & Lateral passage \\
\hline$A m N$ & Antennal motor nerve & $\mathrm{mACT}$ & Median antenno-cerebral tract \\
\hline AN & Antennal nerve & $\mathrm{mCal}$ & Median calyx \\
\hline ASTIT & ASTi tract & MdN & Mandible nerve \\
\hline bR & Basal ring & Me & Medulla \\
\hline $\mathrm{CA}$ & Corpora allata & $\mathrm{mL}$ & Medial lobe \\
\hline $\mathrm{CB}$ & Central body & $\mathrm{mPC}$ & Median protocerebrum \\
\hline $\mathrm{CBI}$ & Lower division of central body & mPed & Median pedunculus \\
\hline CBu & Upper division of central body & MXMT & Maxillar midline tract \\
\hline $\mathrm{CC}$ & Corpora cardiaca & $\mathrm{MxN}$ & Maxillar nerve \\
\hline $\mathrm{Col}$ & Collar & $\mathrm{N}$ & Neck of the pedunculus \\
\hline D02, D03 & Glomeruli 2 and 3 , respectively, innervated by T4 & No & Noduli \\
\hline $\mathrm{DC}$ & Deutocerebrum & OT & Optic tubercle \\
\hline DL & Dorsal lobe & orr & Optic tubercle tract \\
\hline $\mathrm{E}$ & Esophagus & PB & Protocerebral bridge \\
\hline EF & Esophageal foramen & Ped & Pedunculus \\
\hline FN & Frontal nerve & $\mathrm{pPC}$ & Posterior protocerebrum \\
\hline G & Glomerulus of the antennal lobe & s & Somata of the inferior anterior soma layer \\
\hline ich & Inner chiasma & SEG & Subesophageal ganglion \\
\hline iPC & Inferior protocerebrum & SPC & Superior protocerebrum \\
\hline IsT & Isthmus tract & $\mathrm{T} 1, \mathrm{~T}_{4}$ & Sensory neurites in tract 1 and tract 4 , respectively \\
\hline $\mathrm{KC}$ & Kenyon cells & $T 4-6$ & Sensory tracts 4 to 6 \\
\hline La & Lamina & To11 & Antennal sensory tract 611 \\
\hline IACL & Lateral accessory lobe & TC & Iritocerebrum \\
\hline LbN & Labial nerve & vcbnT & Ventral cell body neuron tract \\
\hline LbrN & Labral nerve & $\mathrm{VL}$ & Vertical lobe \\
\hline ICal & Lateral calyx & VLT & Oblique vertical tobe tract \\
\hline $\mathrm{LH}$ & Lateral horn & $\gamma L$ & $y$-lobe \\
\hline lip & Lip of the calyx & & \\
\hline
\end{tabular}


et al., 1996; Homberg et al., 1999). In antennal lobes of moths AST colocalizes with GABA and other peptides (Berg et al., 2007, 2009). Co-release with GABA is likely related to the inhibitory function of AST.

AST-immunoreactivity and the presence of AST limited to specific neuropils has been reported in the developing and adult bee brain (Gräbner et al., 2005; Böhm et al., 2009), but so far no detailed immunohistochemical analysis of the adult brain has been undertaken. In this study we therefore investigated the immunohistochemistry for AST in the honeybee brain and complemented this with double labeling for GABA. We found very pronounced staining in all neuropils, including sensory, central, and motor systems. Given the distinct labeling that we found in the antennal lobe and in olfactory areas of the mushroom bodies, we paid particular attention to the olfactory system and analyzed the detailed branching within olfactory glomeruli by counterstaining for sensory neuron terminals. Our results give a comprehensive overview of ASTir neurons in the adult honeybee brain.

\section{MATERIALS AND METHODS}

\section{Animals}

Honeybees (Apis mellifera) were maintained outdoors in summer, and in a climatic chamber with controlled heat, light, and humidity in winter. We ensured that all bees were foragers by collecting bees individually from a sugar-water baited feeder positioned close to the hive.

\section{Primary antibodies used}

We used a polyclonal rabbit anti-AST antiserum kindly provided by H.J. Agricola (Friedrich Schiller University Jena, Germany). The antiserum was raised against DipAST I conjugated to bovine thyrogloblulin (Vitzthum et al., 1996). Dip-AST I has been also termed DippuAST 7 (Woodhead et al., 1989; Bendena et al., 1999) and exhibits the amino acid sequence APSGAQRLYGFGL-NH antiserum was shown to bind to allatostatins with a $G, A$, or $S$ as the posttyrosyl residue, although it was two orders of magnitude less sensitive to the peptides with the $A$ or the $S$ in a competitive enzyme-linked immunosorbent assay (ELISA) (Vitzthum et al., 1996). In the honeybee at least five peptides sharing the common C-terminal pentapeptide sequence $-Y X F G L-\mathrm{NH}_{2}$ have been detected by mass spectrometry, three of them with $S$ as posttyrosyl residue (Audsley and Weaver, 2006; Hummon et al., 2006; Predel and Neupert, 2007; Brockmann et al., 2009). Thus, the Dip-AST I antiserum binds to at least the three honeybee AST isoforms sharing the -YSFGL-NH ${ }_{2}$ sequence.

Separate preadsorption controls were carried out for AST I and AST VI (Bachem, King of Prussia, PA), the latter sharing a posttyrosyl serine residue with three of the honeybee ASTs. The peptides were dissolved in distilled water and diluted with phosphate-buffered saline with the addition of $0.2 \%$ Triton $X-100$ (PBSTX 0.2) with $0.2 \%$ bovine serum albumin (BSA) and $0.02 \% \mathrm{NaN}_{3}$ to a final concentration of $10^{-4} \mathrm{~mol} \mathrm{~L}^{-1}$ and antibody was added to a final dilution of 1:5,000. After 2 hours of incubation at room temperature (RT), brains, processed as below, were added and the procedure was continued as described below. Both peptides independently and completely abolished staining, whereas positive controls performed in parallel displayed bright staining.

We used a polyclonal rabbit anti-GABA antiserum (4TB) kindly provided by Dr. H. Dircksen (University of Stockholm, Sweden). The production and specificity of the antiGABA antiserum has been characterized previously (Homberg et al., 1999). It was raised against GABA coupled to BSA, hemoglobin, and poly-L-Lysine as carrier proteins. In the locust the antiserum has revealed the distribution of GABAir neurons, being identical to the distribution of neurons immunoreactive for the GABA-synthesizing enzyme glutamic acid decarboxylase. Preadsorbing the 4TB antiserum with $2010^{-6} \mathrm{~mol} \mathrm{~L}^{-1}$ glutamate-glutaraldehyde complexes only slightly reduced the staining intensity (Homberg et al., 1999).

In control experiments on honeybee brains, staining with the 4TB anti-GABA antiserum was abolished after preadsorbing $2 \mathrm{~mL}$ of antibody solution as above with 10 $\mu \mathrm{L}$ of a GABA-glutaraldehyde-thyroglobulin complex (kindly provided by $\mathrm{H}$. Dircksen). Positive controls on honeybee brains yielded a staining pattern similar to that described previously (Schäfer and Bicker, 1986). Omitting the primary antisera resulted in no staining of any specific brain structures.

\section{AST-immunostaining in whole mounts}

The study is based on a total of 157 immunostained whole-mount brains. For immunohistochemistry, bees were quickly immobilized on ice and the antennae were cut close to the basal articulation. The bees were perfused with the fixative by injecting $4 \%$ paraformaldehyde in PBS $(\mathrm{pH} 7.4,5.7 \mathrm{~g}$ sucrose $/ \mathrm{L}$ added to the fixative to adjust osmolarity; all chemicals were purchased from Sigma, St. Louis, MO, except otherwise stated) into the thorax until drops of fixative extruded from the cut ends of the antennae. This procedure ensures quick fixation and avoids mechanical tissue distortion before fixation. The brains were then dissected in fixative solution and postfixed until a total fixation period of 2 hours at RT Next, brains were repeatedly washed in PBS with the addition of $0.2 \%$ PBSTX 0.2 for at least 12 hours. Brains were preincubated at $4^{\circ} \mathrm{C}$ for at least 12 hours in blocking buffer (PBS containing $0.5 \%-1 \%$ Triton $X-100,0.2 \%-1 \%$ 
BSA, and $0.02 \% \mathrm{NaN}_{3}$ ). Rabbit anti-AST was applied at $1: 2,000-1: 10,000$ in PBSTX 0.2 with $0.2 \%$ BSA and $0.02 \%$ $\mathrm{NaN}_{3}$ for 6-8 days at RT. All incubations and washing steps were done with constant gentle agitation.

After incubation with the primary antiserum the brains were washed at least 5 times at RT for 2 hours each. Secondary goat antirabbit antibodies conjugated to Alexa 488 , Alexa 543, or Alexa 633 (Invitrogen, Carlsbad, CA) or $F\left(\mathrm{ab}^{\prime}\right)_{2}$ fragments of goat anti-rabbit antibodies conjugated to Cy3 (Jackson ImmunoResearch Europe, Newmarket, UK) were used at dilutions of 1:500-1:1,000 in PBSTX 0.2 with $0.2 \%$ BSA and $0.02 \% \mathrm{NaN}_{3}$ for $6-8$ days at RT. Subsequently, the brains were washed repeatedly in PBS, dehydrated in an ethanol series $(50 \%, 70 \%, 90 \%$, $98 \%, 100 \%, 100 \%, 10-20$ minutes each), cleared in xylene ( $2 \times 3$ minutes), and mounted in DPX (Sigma-Aldrich Chemie, München, Germany) between two coverslips spaced by custom-made metal frames of $0.6 \mathrm{~mm}$ thickness in order to enable inspection from both the anterior and posterior aspect.

Some brains were reoriented to obtain horizontal or sagittal scans and to clarify neurite branching patterns in areas that were otherwise difficult to visualize. To that aim, mounted and preliminary scanned specimen were reimmersed in xylene overnight, removed from the coverslips, and then cut into two pieces either in a medial horizontal or in a medial sagittal plane. The brains were subsequently remounted as above.

\section{Double staining of AST and antennal afferents or protocerebral neuropils}

In order to analyze the relationship between ASTir neurons and olfactory receptor neuron axons, antennal afferents were stained as described elsewhere (Galizia et al., 1999). Briefly, cooled bees were harnessed in plastic holders and the antennae were immobilized in a vertical position with Eicosan. A well of Vaseline was built around the antennae and filled with $2 \%$ neurobiotin (Vector Laboratories, Burlingame, CA) in distilled water. The antennae were cut at the base of the flagellum or at the pedicel and the well was sealed with Vaseline. Animals were kept in a moist dark chamber for 4-12 hours at RT, after which the well containing the neurobiotin solution was carefully removed. Next, bee brains were fixed with $4 \%$ paraformaldehyde, dissected, and labeled for AST as described above. To detect neurobiotin in the sensory fibers, avidinAMCA (Vector Laboratories) or streptavidin-Cy3 (Jackson ImmunoResearch Europe), at a dilution of 1:500, was applied together with the secondary antiserum.

In order to relate ASTir structures to projection areas of other neurons we combined mass fills of neuron populations (Sachse and Galizia, 2002) with AST immunostaining. Bees were caught and harnessed as above. A window was cut in the frontal head capsule and glands and trachea were displaced from the target area to access the brain. Then the tip of a glass capillary coated with Alexa 547 Dextran MW 10,000 or Alexa 647 Dextran MW 10,000 (all from Invitrogen) was manually inserted into the tissue for 2 seconds. The capillary was removed, the excised piece of cuticula was repositioned, and the head capsule was sealed with Eicosane. The dye was allowed to travel for 4-12 hours in the living brain. Thereafter, brains were fixed and immunostained as described above.

Specifically, we labeled olfactory projection neurons from the mushroom bodies or other mushroom body extrinsic neurons by placing the crystal at their entry to the mushroom bodies between the calyces. Similarly, in other animals we inserted the crystal into the mushroom body (MB) vertical lobes to identify vertical lobe extrinsic neurons.

\section{GABA- and AST-double-staining in whole mounts}

Double labeling using GABA and AST antisera was done in a sequential manner, since both antibodies were derived from rabbit. In a first round, the GABA antiserum was diluted 1:30,000 and applied as described above, followed by staining with an excess concentration of Alexa 488 conjugated $F\left(a b^{\prime}\right)_{2}$ fragments of goat antirabbit antisera (Invitrogen, diluted $1: 200$ in PBS- $0.2 \%$ TX including $0.2 \% \mathrm{BSA}$ and $0.02 \% \mathrm{NaN}_{3}$ and applied for 5 days at RT). After extensive washing for at least $5 \times 2$ hours and overnight, in a second round the AST antiserum was applied as above and specific binding was detected with a secondary goat antirabbit antiserum coupled to Alexa 633 (Invitrogen). After this treatment, all GABA-immunoreactive (GABAir) neurons were labeled with Alexa 488 and may also be labeled with Alexa 633, while ASTir neurons that are not GABAir were labeled with Alexa 633 only. The procedure does not allow for differentiation between GABAir neurons that have only GABA and those that coexpress GABA and AST because secondary antibody of the second round may bind to GABA antibody binding sites that were left unbound in the first round. However, neurons that exclusively are ASTir but devoid of GABA were only labeled in the second round.

\section{Confocal data analysis}

Whole mounts were scanned using a Zeiss LSM 510 NL confocal microscope (Carl Zeiss, Jena, Germany). In confocal mode we used the following laser lines: 488 , 543,633 and appropriate filter combinations. However, images slightly deteriorate with increasing depth. We therefore also used 2-photon excitation, a technique that allows deeper penetration into the tissue, while maintaining the spatial resolution of confocal microscopy. We used $750 \mathrm{~nm}$ to excite AMCA and DAPI. Stacks were 

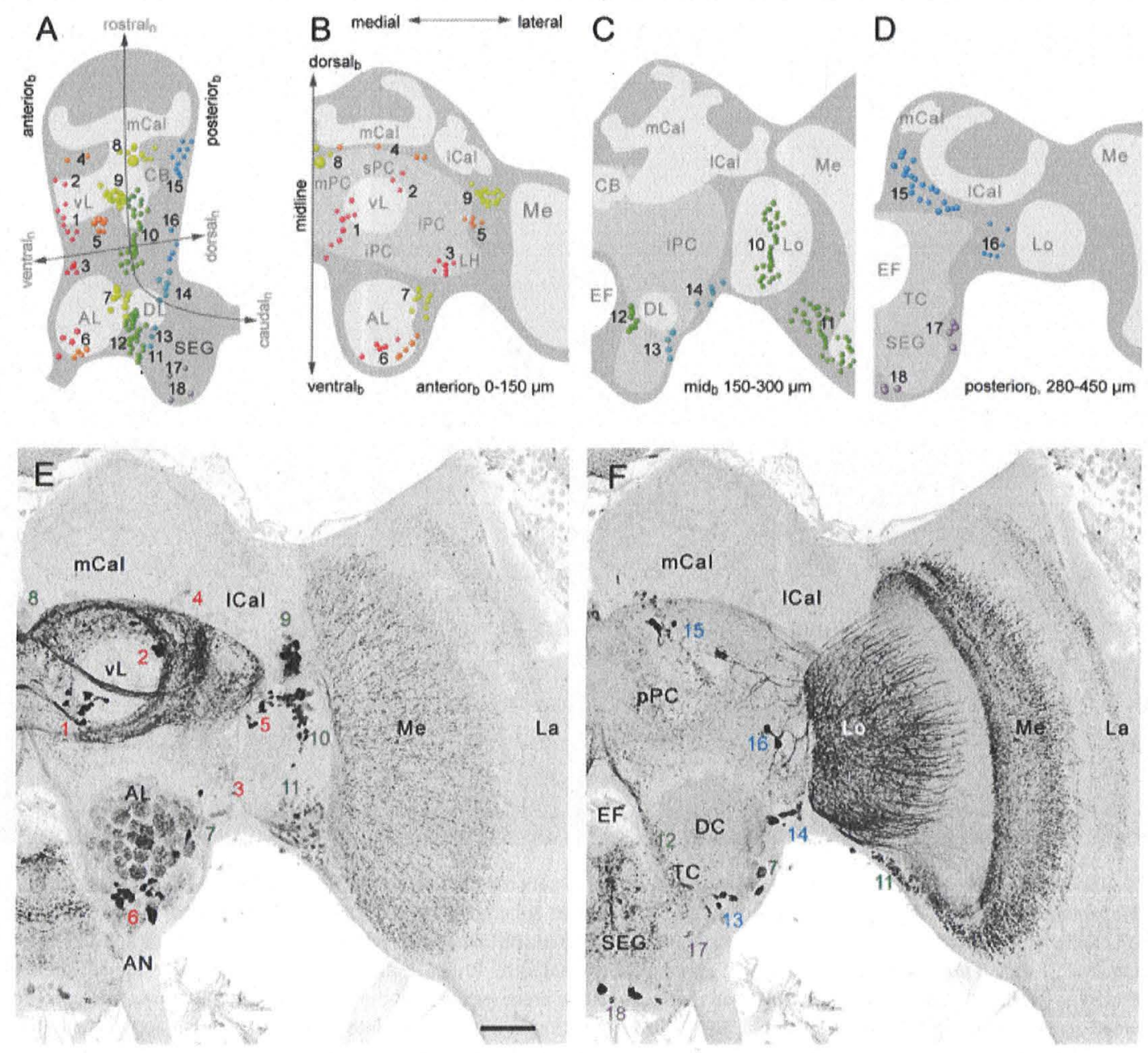

Figure 1. Summary of ASTir soma groups. A-D: Schematic drawings of ASTir soma groups in a sagittal and three consecutive frontal layers. Throughout the text we refer to body axis only (subscript $b$ only in this figure). Here we give the neuraxis for comparison (subscript $n$ in A) and shown by the gray rostrocaudal line in the sagittal scheme. The neuraxis is bent upwards between the subesophageal ganglion and the rostral pole, thus changing its direction with respect to the body axis. In the sagittal drawing at the left (A), anterior is to the left and posterior to the right. Dorsal is up and ventral down. The three frontal schemes of the left hemisphere are drawn as layers at 0-150 $\mu \mathrm{m}$ (B), 150-300 $\mu \mathrm{m}$ (C), and 300-450 $\mu \mathrm{m}$ (D) distance from the anterior surface of the protocerebrum. Colors of soma groups in a rainbow color sequence indicate increasing distances from the anterior surface. Soma diameters are not to scale. E: Transparent projection of the anterior half $(0-300 \mu \mathrm{m})$ of an AST labeled whole mount. ASTir neuropils and soma groups are apparent. ASTir soma groups are labeled with numbers as in A. F: Projection as in E, but of the posterior half, seen from posterior and mirrored for ease of comparison. Unless otherwise stated, all subsequent figures show frontal projections from the left hemisphere, as in E. For abbreviations, see list. Scale bar $=100 \mu \mathrm{m}$ in $\mathrm{E}$ (applies to F).

scanned using a $10 \times 10.45$, or a $20 \times / 1.0$ water immersion objective. To obtain high-magnification scans for measuring of neuritic dimensions, we used a $63 \times / 1.4$ oil objective.

Images were analyzed and visualized using the Zeiss LSM image browser or AMIRA 5.2.1 (Visage Imaging, Berlin, Germany). Several single optical sections were superimposed in maximal intensity mode to obtain projections of optical slices of intermediate thickness. Transparent projections (e.g., Fig. 1) were used to visualize thick scans. Here, objects in deeper layers are faded, giving a better rendering of 3D information. The projections were inverted and further processed to adjust brightness and contrast using Adobe Photoshop CS2 software (Adobe Systems, San Jose, CA). Schematic drawings were generated with Adobe Illustrator CS4. Movies for supporting material were created using ImageJ (NIH, Bethesda, MD) and were labeled with the aid of a custom-written plug-in.

Because of the small number of stained neurons in most cell groups we relied on manually counting stained 
cell bodies from the confocal stacks. If not stated otherwise, numbers are based on counts in $4-8$ brains and represent the approximate minimum in one brain hemisphere.

\section{Nomenclature}

Throughout this article we use body axes as a reference, and not neuraxes, with the exception of Figure $1 \mathrm{~A}$, where we show both in order to clarify them. In the
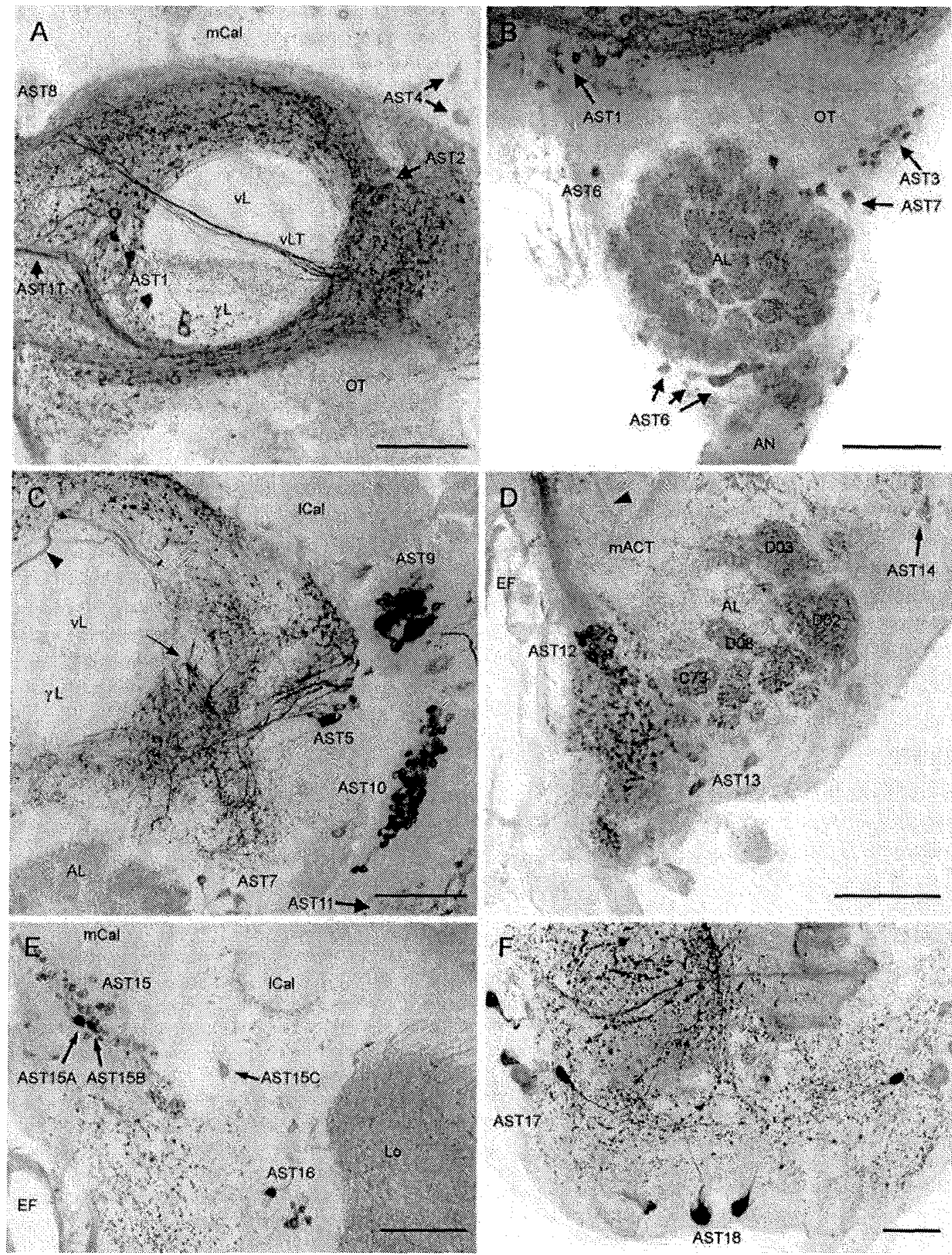

Figure 2 
honeybee the longitudinal neuraxis, a virtual straight line connecting the brain neuromeres and all ganglia of the ventral nerve chord, is bent dorsally between the subesophageal ganglion and the brain in a smooth curvature of $90^{\circ}$ (Fig. 1A). Therefore, in the head, and referring to the body axis, the neurally anterior (rostral) ocelli are at the top of the brain or dorsal, the neurally ventral part of the protocerebral neuromere are the front of the brain or anterior, the neurally posterior part is the base of the brain or ventral, and the neurally dorsal parts of the brain neuromeres are the back of the brain or posterior.

Where not indicated otherwise, naming of brain structure and tracts followed the previous seminal articles (Suzuki, 1975; Mobbs, 1982a; Arnold et al., 1985; Mobbs et al., 1985; Rehder, 1988; Strausfeld, 2002). Specifically, each MB of the honeybee consists of two calyces and two lobes, which are connected by the axons of the intrinsic Kenyon cells running in the pedunculi. The anteriorly directed $\alpha$-lobe (Mobbs, 1982a,b) has also been termed the vertical lobe. It can further be subdivided into the three dorsal divisions, containing type I Kenyon cell axons, and the $\gamma$-lobe, containing type II Kenyon cell axons (Strausfeld, 2002). We use the term vertical lobe when referring to the three dorsal divisions of the vertical lobe and the $\gamma$-lobe as a composite neuropil. We use the term medial lobe for the medially directed lobe of the MB, which is also known as the $\beta$-lobe.

To provide information on positions of structures within the brain we measured the distances from the anterior surface of the brain, which we defined as the most frontal plane of the antennal lobes and the vertical lobes.

\section{RESULTS}

\section{ASTir somata in the honeybee brain}

A clear staining against AST was visible in almost all neuropils of the honeybee brain, comprising cell bodies and neurites of various dimensions. We found a total of about 550 ASTir cell bodies, which we sorted into 18 groups in ventral, intermediate, and dorsal parts of the peripheral soma layer of the brain (Figs. 1, 2). We do not call these groups "clusters" because some were loosely packed, and the small-scale position varied across individuals (see below). Even within groups, staining intensity was variable, and also within the denser groups we found unstained somata scattered among the stained ones (Fig. 2). The ASTir neurons projected via bundles of neurites into neuropils of the protocerebrum including the optic lobes, the superior, inferior, median, and lateral protocerebral lobes, the central body (CB) and the MBs (Figs. 3-7). Clear AST staining was also found in somata and the neuropil of the antennal lobes (ALs) in the deutocerebrum (Figs. 8-10), in the tritocerebrum, and in the subesophageal ganglion (SEG) (Fig. 11). The abundance of fine ASTir neurites made it difficult to trace connections of individual neurites to their soma, which we therefore only describe in unequivocal cases.

In the following we first list the 18 ASTir soma groups (AST1 to AST18) with a description of position, size, and staining intensity, as well as their consistently traceable primary neurites where possible. Numbers are attributed as names in sequence along the anterior to posterior body axis and have no functional implication. A schematic view of soma clusters is given in Figure $1 A-D$ and frontal projections of the brain from the front and from the back are given in Figure 1E,F, respectively. Magnified views of individual soma groups are provided in Figure 2. Next we present a more detailed description of ASTir neuron morphology in the context of each innervated brain structure (Figs. 3-11).

\section{AST1}

These cell bodies were scattered in variable positions in front of the anterior surface of the vertical lobe and within the soma cortex mediodorsaliy and medioventrally

Figure 2. ASTir soma groups in the bee brain in confocal whole-mount projections, from anterior to posterior. A: Vertical lobe area. Soma group 1 (AST1) and its associated tract (AST1T) lie medioventrally next to the vertical lobe (see also somata in B). AST2 lies at the opposite side. A detailed analysis of the vertical lobe is in Fig. 6. AST4 is mainly situated between the MB calyces. AST8 somata are in the midline but in a more posterior plane. B: Antennal lobe area. AST3 is located next to the optic tubercle. AST6 and AST7 are associated with the antennal lobe (see also Fig. 8). Note the vertical lobe tract running obliquely in front of the vertical lobe. C: Lateral protocerebrum area. AST5 somata lie laterally of the vertical lobe. Their neurites (arrow) give rise to the vLT (see A). AST9 and AST10 are located medial to the frontal medulla and anterior of the lobula. Both groups project into the visual system. AST9 also sends collaterals into the protocerebrum. AST11 is located proximal of the ventral medulla. Arrowhead indicates neurites of AST15A-C (see E). D: Dorsal lobe area. AST12 is located medial to the posterior antennal lobe. AST13 is ventral to the dorsal lobe (see Fig. 11). AST14 is laterodorsal to the dorsal lobe and may form a continuous group with AST16. Note that the stained neurite along the mACT (arrowhead) was traced into the SEG and not into the AL. E: Posterior protocerebrum area. Into the group AST15 we included all somata located in the median posterior protocerebrum beneath the MB calyces. The large neurons AST15A, 15B, and 15C have prominent primary neurites and arborize in large areas of the brain (see arrowhead in C, Figs. 5, 6). AST16 is located lateroventral to AST15. F: Subesophageal ganglion area. AST17 is located in the lateral soma layer, AST18 is located in the medial soma layer. For abbreviations, see list. Scale bars $=100 \mu \mathrm{m}$ in A-E; $50 \mu \mathrm{m}$ in $\mathrm{F}$. 



Figure 3. Consecutive projections of the lateral protocerebrum showing strong staining of the ring neuropil and the innervation pattern of groups AST9 and AST10 in the optic lobes. The projections, $30 \mu \mathrm{m}$ each, are at increasing depth from the frontal surface of the brain as indicated in $\mu \mathrm{m}$ in the upper-right corner of each frame. See also Supporting Movie SM2. Fibers in the optic tubercle tract are not immunoreactive $(A, B)$. AST9 is formed by $\approx 60$ somata, located anteriorly between medulla and lateral protocerebrum (A-D). Neurites travel medioventrally first (arrow in C), and then dive posteriorly (AST9N in D,E). At least part of the bigger neurites bifurcate to contribute to the intermingled mesh of ASTir neurites in the central protocerebrum (AST9N in E). The smaller neurites turn laterally to innervate the medulla $(F)$. Group AST10 contains $\approx 75$ neurons, located ventral of AST9 $(A-C)$. Their neurites travel posteriorly and spread into a fan innervating the entire lobula (labeled with AST10N in D-I). From the dense meshwork in the most distal layer of the lobula, large fibers enter the inner chiasma toward the medulla $(F-I)$. The lobula receives additional AST supply by a faintly stained tract with unknown origin in the inferior posterior protocerebrum (arrowhead in C and D). Primary neurites of AST11 somata (in B) are outside the frame and only faintly stained. They most likely also enter the medulla. For abbreviations, see list. Scale bar $=50 \mu \mathrm{m}$ in A (applies to all).

of the vertical lobe, within $40 \mu \mathrm{m}$ from the anterior surface of the brain. The group comprised about 14 clearly and reliably stained neurons, with short diameters of about $9 \mu \mathrm{m}$, near the lobe, and additionally up to 10 less reliably stained somata near the esophageal foramen. The AST1 group was found in a territory clearly distinct 

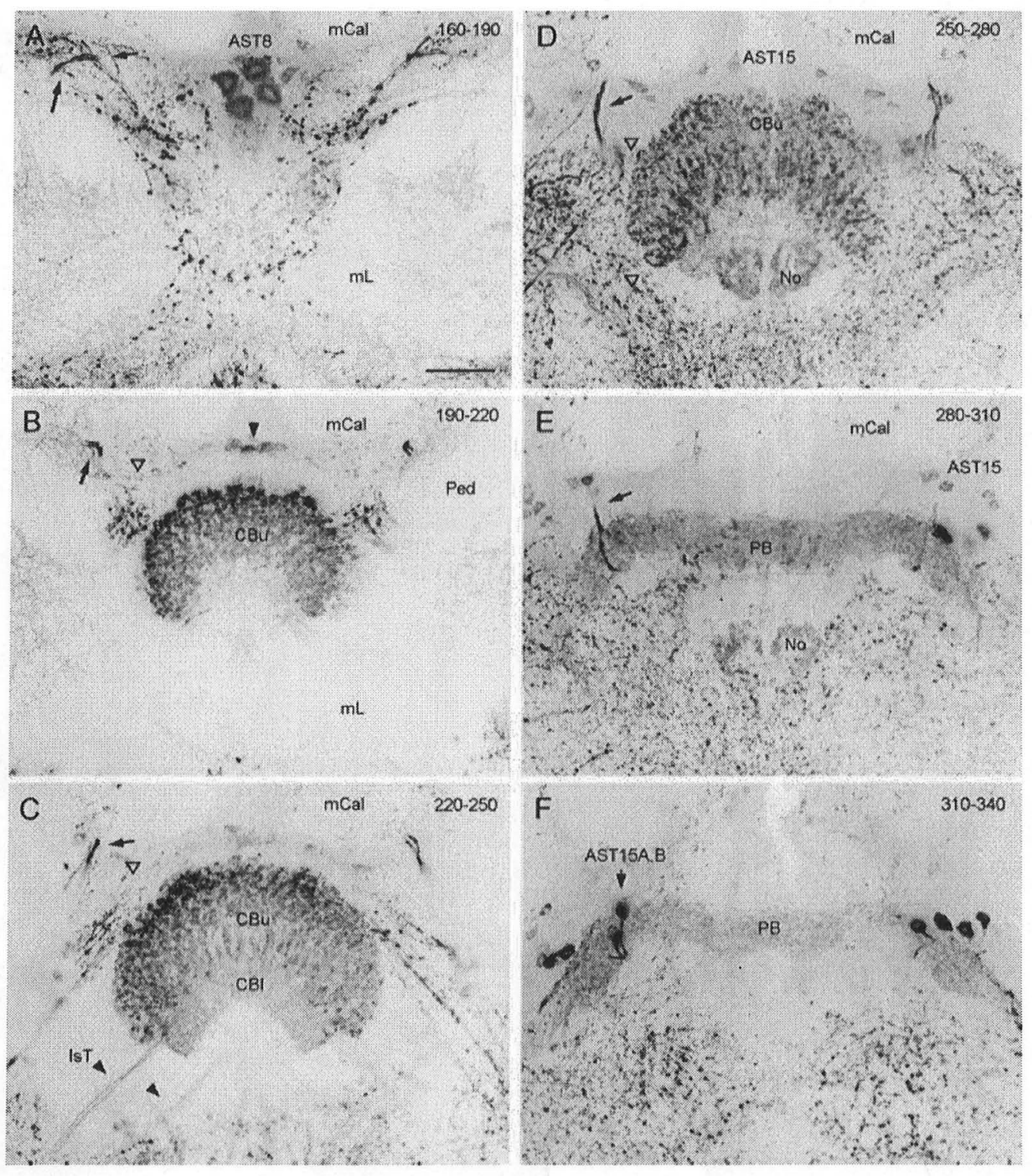

Figure 4. Central complex, consecutive projections, 30- $\mu \mathrm{m}$ thickness each (A-F). Depth of the optical slice is indicated in $\mu \mathrm{m}$. The central body is densely innervated by ASTir profiles. The anterior part and the posterior part of the upper division show stronger immunoreactivity than the lower division (B-D). The noduli and the protocerebral bridge are also immunoreactive (D-F). Note the labeling of fibers in the isthmus tracts (arrowheads in C), connecting the central body to the lateral accessory lobes. Neurites of smaller somata in the AST15 group and other groups likely contribute to the staining in the central complex. The arrows in A-F point to neurites of the AST15A, 15B, and $15 \mathrm{C}$ neurons, which clearly pass the central complex without invading any of its parts (see Fig. 5). AST8 somata send primary neurites posteriorly without entering the central body (A and arrowhead in B). Note that the path of the MACT is indicated with open triangles in B-D and contains no ASTir fibers. For abbreviations, see list. Scale bar $=50 \mu \mathrm{m}$ in A (applies to all).

from that occupied by the groups $A 1, A 2$, and $A 5$ of $M B$ extrinsic neurons (Rybak and Menzel, 1993). Consistently, AST1 neurons did not enter the MBs (Supporting Movies SM1, SM3, SM4). The primary neurites of the more dorsal somata of AST1 were thin $(<1 \mu \mathrm{m})$ and projected medially. At least some of them bifurcated and sent one branch below the vertical lobe. The other branches fused to form a horizontal tract, which crossed to the contralateral hemisphere at a depth of about $50 \mu \mathrm{m}$ from the anterior surface of the brain (Fig. 2A). Contralaterally, the tract spread below the $\gamma$-lobes to enter the anterior lateral protocerebrum. Here the ramifying fine and varicose neurites could not be traced further. AST1 somata that lay more ventrally near the esophageal 

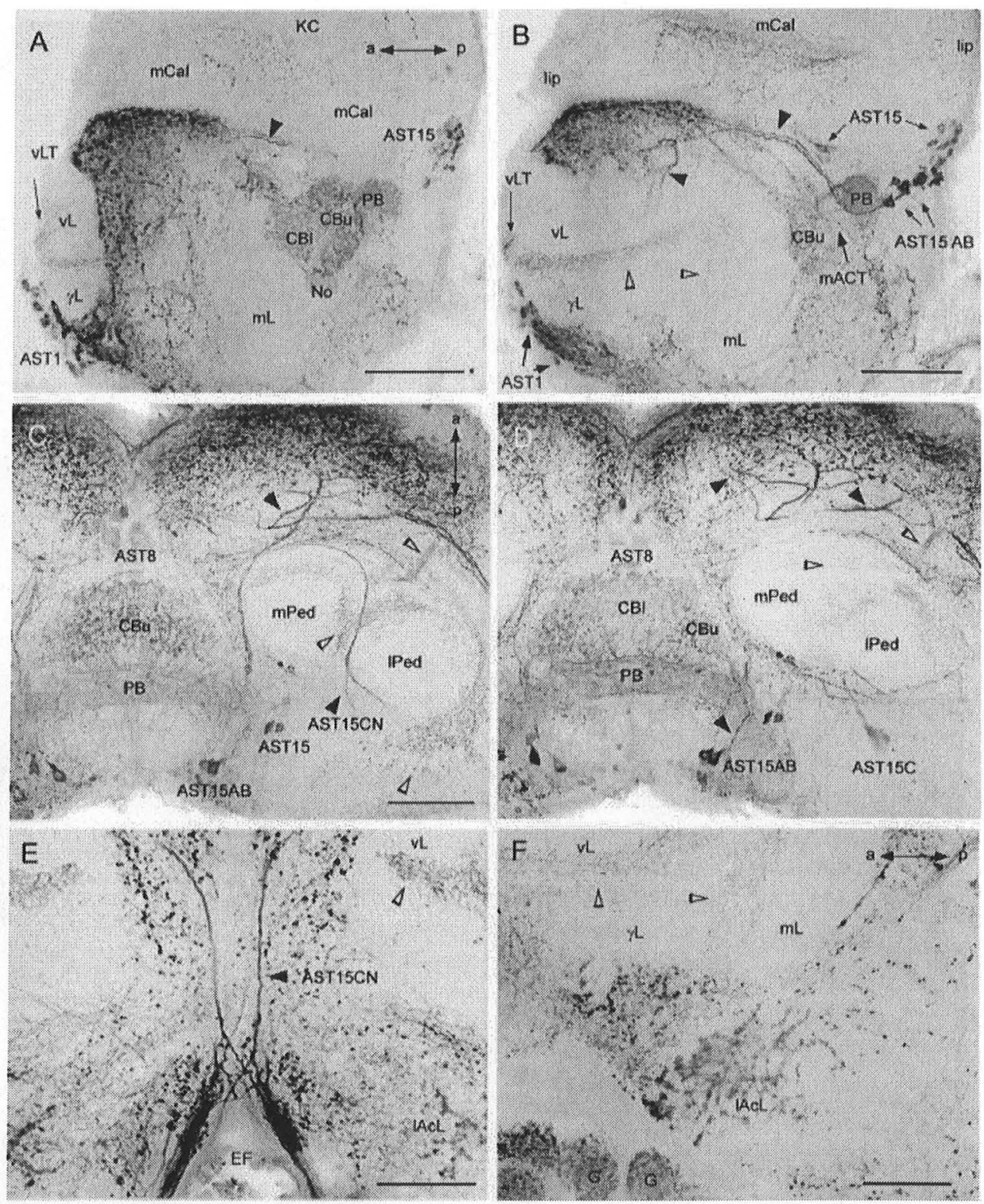

Figure 5. Protocerebral neuropils and long-range innervation by AST15A, 15B, and 15C. A,B: Sagittal sections, A is slightly lateral to the midline, $B$ is more lateral than $A$. Anterior is to the left and posterior to the right. Note the separation of the upper and lower division of the central body and immunoreactivity in the protocerebral bridge and in the noduli in A. The AST15A, AST15B neurons send their primary neurites past the protocerebral bridge into the anterior ring neuropil around the vertical lobe (closed arrowheads in A-E). The vertical lobe only shows immunoreactive neurites in ventral divisions and in the $\gamma$-lobe (indicated by open arrowheads, see also Fig. 6). The staining in the medial lobe is also indicated by open arrowheads. Note that the mACT is devoid of ASTir fibers. The anterior ring neuropil surrounding the vertical lobe and the oblique vertical lobe tract is visible to the left because the section is slightly oblique. C,D: Horizontal slices, $40 \mu \mathrm{m}$ each, showing AST15A, B, and C somata and their projections to the ring neuropil in a plane above the vertical lobes. The neuron AST15C sends a neurite between the pedunculi of the lateral and medial calyx. The full trajectory toward the brain hemispheres is not visible in $C$ but continued in D (closed arrowheads). Staining within the pedunculi comes from AST3 and is marked with open arrowheads. (see Figs. 6, 7). E: Frontal view of the median protocerebrum in a plane slightly anterior of the central body. Neurites of the AST $15 \mathrm{C}$ neurons cross the midline to supply the contralateral hemisphere. The strong staining around the esophageal foramen mainly originates from AST12 somata (see Fig. 2D). F: Sagittal section, 5- $\mu \mathrm{m}$ thick, showing staining in the lateral accessory lobe. ASTir neurites form a cloud of large, vesicular structures that are difficult to trace as individual neurites. These neurites are connected to the central body via the isthmus tract (see Fig. 4). They could not be traced to an identified soma group. Note the layers of labeled fibers (open arrowheads) in the three dorsal divisions of the vertical lobe and in the $\gamma$-lobe of the MB. For abbreviations, see list. Scale bars $=100 \mu \mathrm{m}$ in A,B; $50 \mu \mathrm{m}$ in C,E,F (applies to D). 



Figure 6. MB lobes and pedunculus of the left hemisphere. Consecutive projections, $30 \mu \mathrm{m}$ each, of the anterior part of the MB. AST1 neurons contribute to the ventral ring neuropil and send collaterals through the central anterior protocerebrum to the contralateral hemisphere (to the left). ASTir neurons are embedded in the ring neuropil. Note the dense meshwork in the ventral division of the vertical lobe above the $\gamma$-lobe (A). Neurites from AST5 travel toward the ring neuropil and project anterodorsally and subsequently form the oblique vertical lobe tract that crosses the vertical lobe (arrowheads in $\mathrm{A}$ and $\mathrm{B}$ ). The tract is barely visible in $\mathrm{A}$ but apparent in Fig. $2 \mathrm{~A}$, which shows a more superficial slice. Large neurites from AST15A, B, C (see Fig. 5) are visible at several depths (asterisks in B,C,D-F). The AST3 group is located in the soma layer beneath the optic tubercle at a depth of $30 \mu \mathrm{m}$ from the anterior surface of the vertical lobe (arrow in A). Minute faintly stained neurites leave AST3 and dive dorsoposteriorly to enter the MBs between the calyces (arrows labeled $60 \mu \mathrm{m}$ in B, 90 $\mu \mathrm{m}$ in $\mathrm{B}, \mathrm{C}, 120 \mu \mathrm{m}$ in C,D, $150 \mu \mathrm{m}$ in D,E; see also Supporting Movie SM3). Here the neurites take two directions. Collaterals head toward the pedunculus necks and form a dense U-shaped meshwork of fine fibers in the inner ring tracts of both calyces and subsequently supply the lobes. The other population directly enters the pedunculus $(D, E)$ also contributing to the dense meshwork in the medial lobe and in the vertical lobe at the border to the $\gamma$-lobe. For abbreviations, see list. Scale bar $=50 \mu \mathrm{m}$ in A (applies to all). 

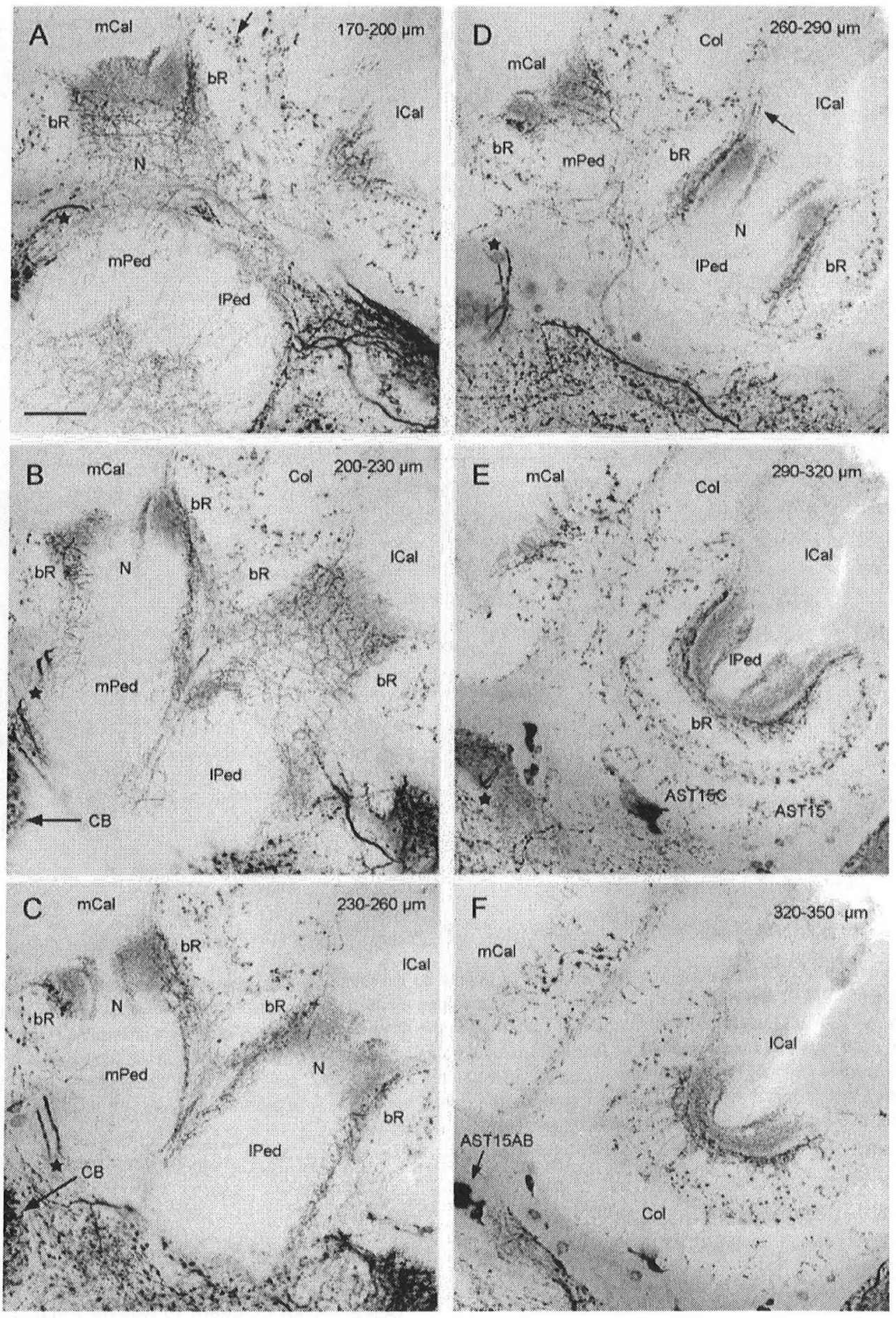

Figure 7. MB pedunculi and calyces in an order approximately corresponding to an overlapping continuation of Fig. 6, but from a different animal. Consecutive projections, $30 \mu \mathrm{m}$ each. The branching of AST3 neurites, which enter the pedunculus and lobes, and those entering the calyces through the basal ring is visible in A (compare with Fig. 6; see also Supporting Movie SM3). The meshwork in the inner ring travels through the necks into the calyces. From the basal ring, collaterals extend toward the outer ring tract of the calyx where they form bleb-like structures (arrows in B,D). The foggy staining in the inner neck likely consists of minute collaterals of the basal ring neurites and descends through a U-shaped tube in a peripheral layer of both pedunculi $(E, F)$. Stars in A-E indicate neurites of the AST15A, 15B, 15C neurons. For abbreviations, see list. Scale bar $=50 \mu \mathrm{m}$ in A (applies to all). 

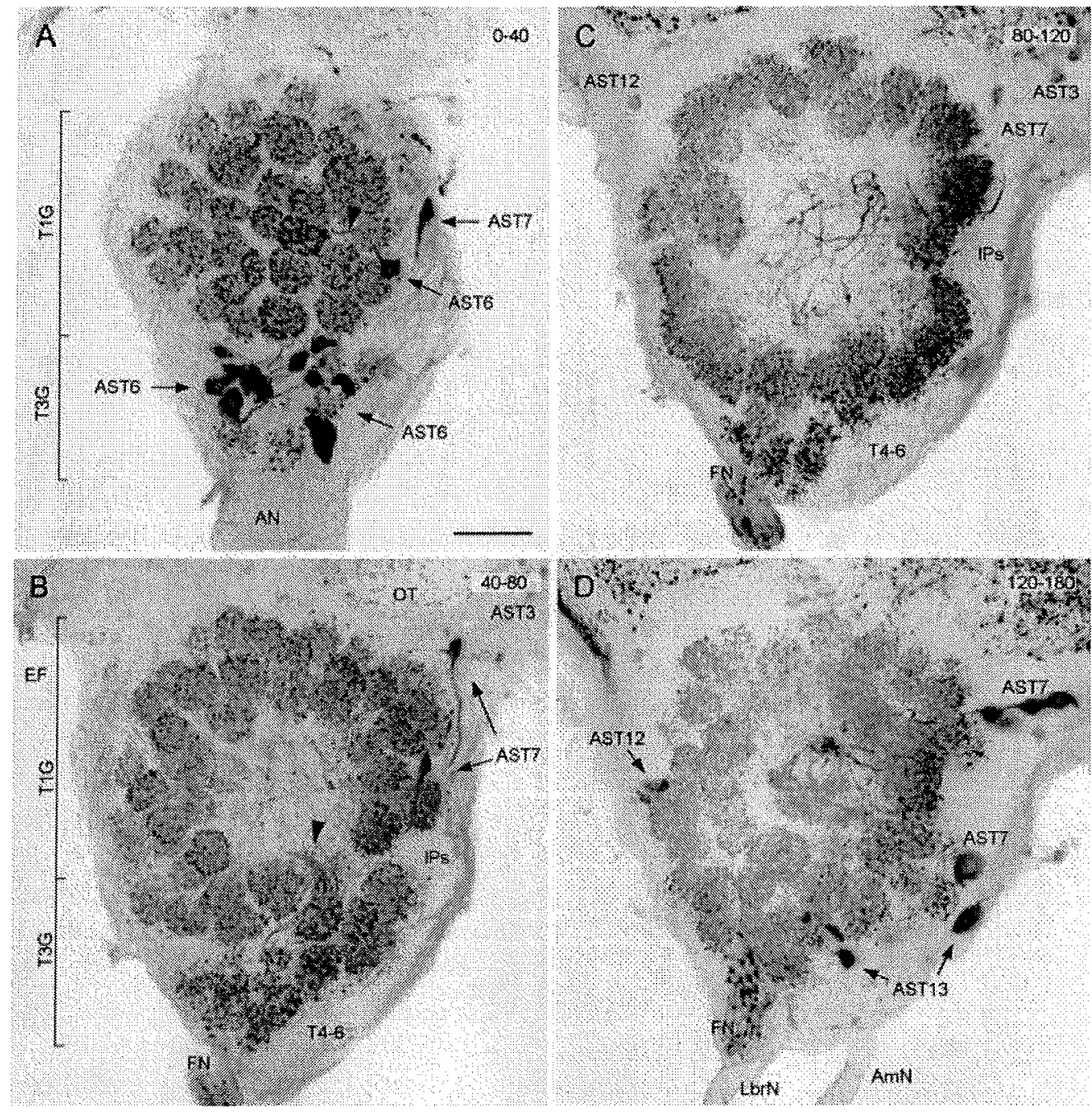

Figure 8. Antennal lobe, consecutive frontal projections of depths as indicated. All glomeruli are densely innervated by ASTir neurites deriving from AST6 $(A, B)$ and AST7 (A-D). T1G and T3G indicate approximate areas with glomeruli innervated by sensory tracts 1 and 3 , respectively. AST3, AST12, and AST13 somata do not contribute to the AL network. Glorneruli of all AL subdivisions (T1, T2, T3, T4) are innervated by ASTir neurites. Note how neurites first enter the central coarse neuropil of the AL traveling between glomeruli (arrowheads in $A, B)$. Some neurites from AST7 use the lateral passage to enter the AL $(B, C)$. See also Supporting Movies SM5, SM6, SM7. Note the strong staining in the frontal nerve (C). The staining above the frontal nerve in $D$ is in the tritocerebrum and originates from AST12. For abbreviations, see list. Scale bar $=50 \mu \mathrm{m}$ in A (applies to all).

foramen did not contribute to the AST1 tract and could not be further traced. At least 10 of the AST1 neurons were not GABAir (Fig. 10C).

\section{AST2}

This small group of about six clustered somata was located $20 \mu \mathrm{m}$ dorsolaterally of the vertical lobe (Figs. $1 A, B, E, 2 A)$. Their projections could not be traced because they reached into a dense network with many other ASTir neurites. AST2 neurons did not show GABA immunostaining (Fig. 10C).

\section{AST3}

These somata were located below the optic tubercle $40-120 \mu \mathrm{m}$ behind the anterior surface of the brain (Figs. $1 A, B, E, 2 B, 8 B, C)$. About 12 somata and their primary neurites were only faintly stained and followed a route remotely resembling that of the A3v neurons, which form the protocerebral calycal tract (PCT) together with the A3d neurons (Rybak and Menzel, 1993). The neurites of the AST3 neurons dived behind the optic tubercle tract up to where the projection paths of $A 3 v$ and $A 3 d$ PCT neurites converge. From there, AST3 neurites followed a more 

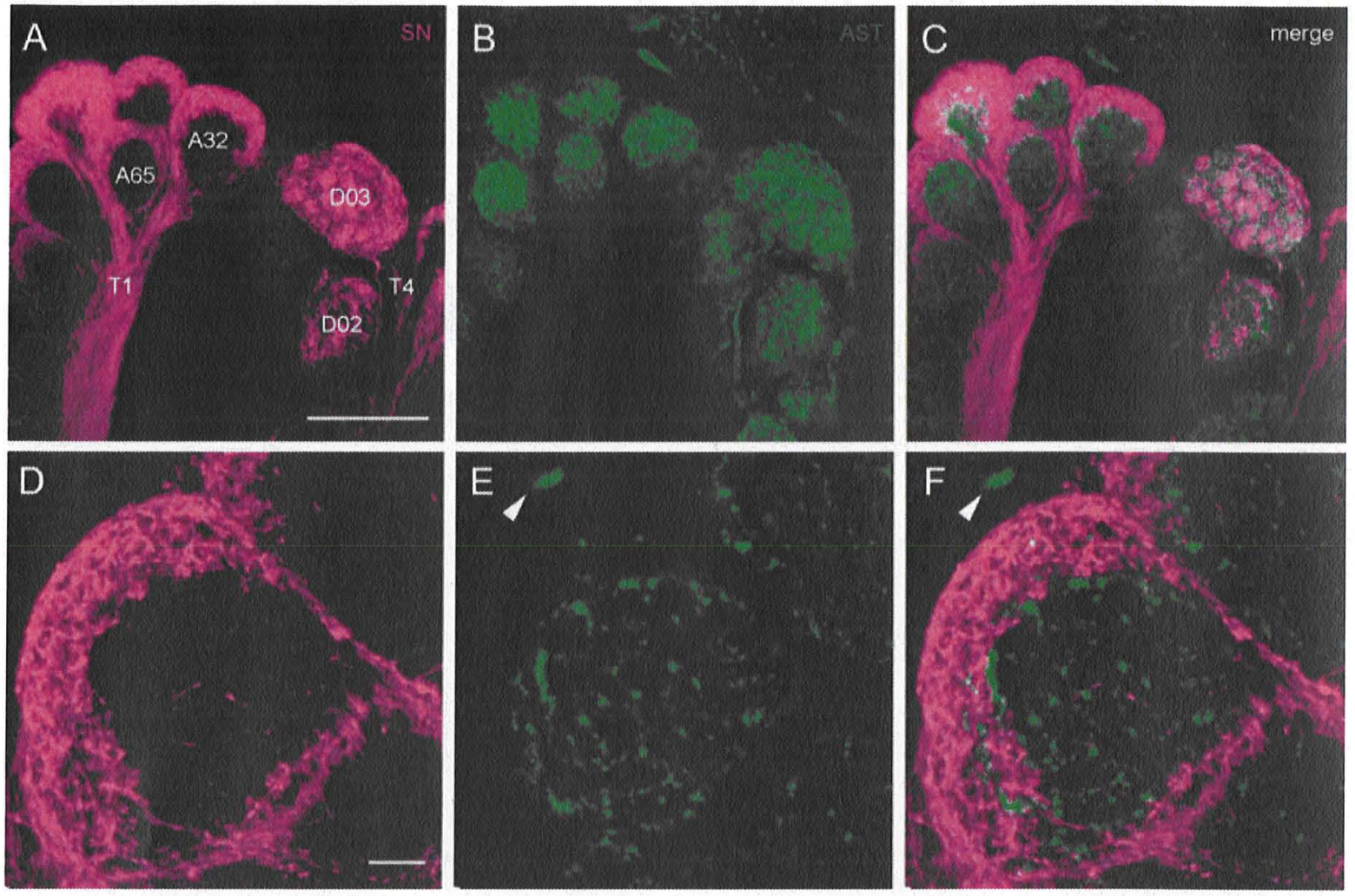

Figure 9. Glomeruli of the antennal lobe. Double staining of antennal sensory neurons (mass fill, A,D) and ASTir (B,E,) merged in C,F. Lateral views, anterior to the left. A-C: $5 \mu \mathrm{m}$ projection of dorsal glomeruli. Sensory neurons of the T1 tract only innervate the cap of glomeruli (e.g., A65, A32), while T4 axons fill their entire glomeruli (e.g., D03, D04). ASTir neurites fill the core in T1 glomeruli and the entire volume in T4 glomeruli. D-E: $0.5 \mu \mathrm{m}$ single optical slice of a glomerulus innervated by T1. ASTir fibers reach marginally into the caps and blebs are concentrated at the border of cap and core. Note also that minor ASTir processes invade the cap, suggesting that synaptic contact is possible between sensory axons and ASTir interneurons. The arrowhead indicates a primary neurite of an AST6 neuron approaching the antennal lobe neuropil from the soma layer. For abbreviations, see list. Scale bars $=50 \mu \mathrm{m}$ in A (applies to B,C); $=10 \mu \mathrm{m}$ in D (applies to E,F).

dorsal and more posterior route than the PCT and reencountered the PCT anteriorly between the calyces of the MBs. They then entered the MB from a horizontal bundle in front of the pedunculi below the calyces and above the vertical lobe. Some branches entered the calyces, some went into the vertical lobe and the medial lobes (Fig. 6, Supporting Movies SM 1, SM3). Our mass fills of the vertical lobe extrinsic neurons revealed that A3v somata were at least $10.5 \mu \mathrm{m}$ in diameter, whereas AST3 somata were about $7.2 \mu \mathrm{m}$, and thus considerably smaller. AST3 somata displayed no GABAir (Supporting Fig. SF1A-F).

\section{AST4}

A group of about seven somata extending in a loose half-ring around the anterior outer rim of the calyces formed AST4 (Figs. 1A,B,E, 2A), including some somata that were found in front of the pedunculi and between the calyces (about $50 \mu \mathrm{m}$ behind the anterior surface). However, no stained primary neurites of AST4 neurons were visible, and therefore no projection could be identified in our preparations.

\section{AST5}

These somata formed a fairly dense and uniform group of about 12 cells positioned in the soma layer of the lateral protocerebrum. In specimens in which AST immunostaining was combined with mass fills of vertical lobe extrinsic neurons, we could locate AST5 somata medially to the A3d neurons that form the PCT (data not shown). It was possible to follow the convoluted tract of AST5 neurites to a large extent (Fig. $2 \mathrm{C}$ ). They entered the lateral protocerebral lobe and crossed PCT neurites medially of the optic tubercle tract. Next, they turned to form a short posterior-anterior segment (60-90 $\mu \mathrm{m}$ from the surface), $60 \mu \mathrm{m}$ further lateral than the vertical lobe lateral border. At least 11 ASTir neurites then emerged near the ventrolateral margin of the vertical lobe, and then passed the vertical lobe forming the oblique vertical lobe tract (vLT) 

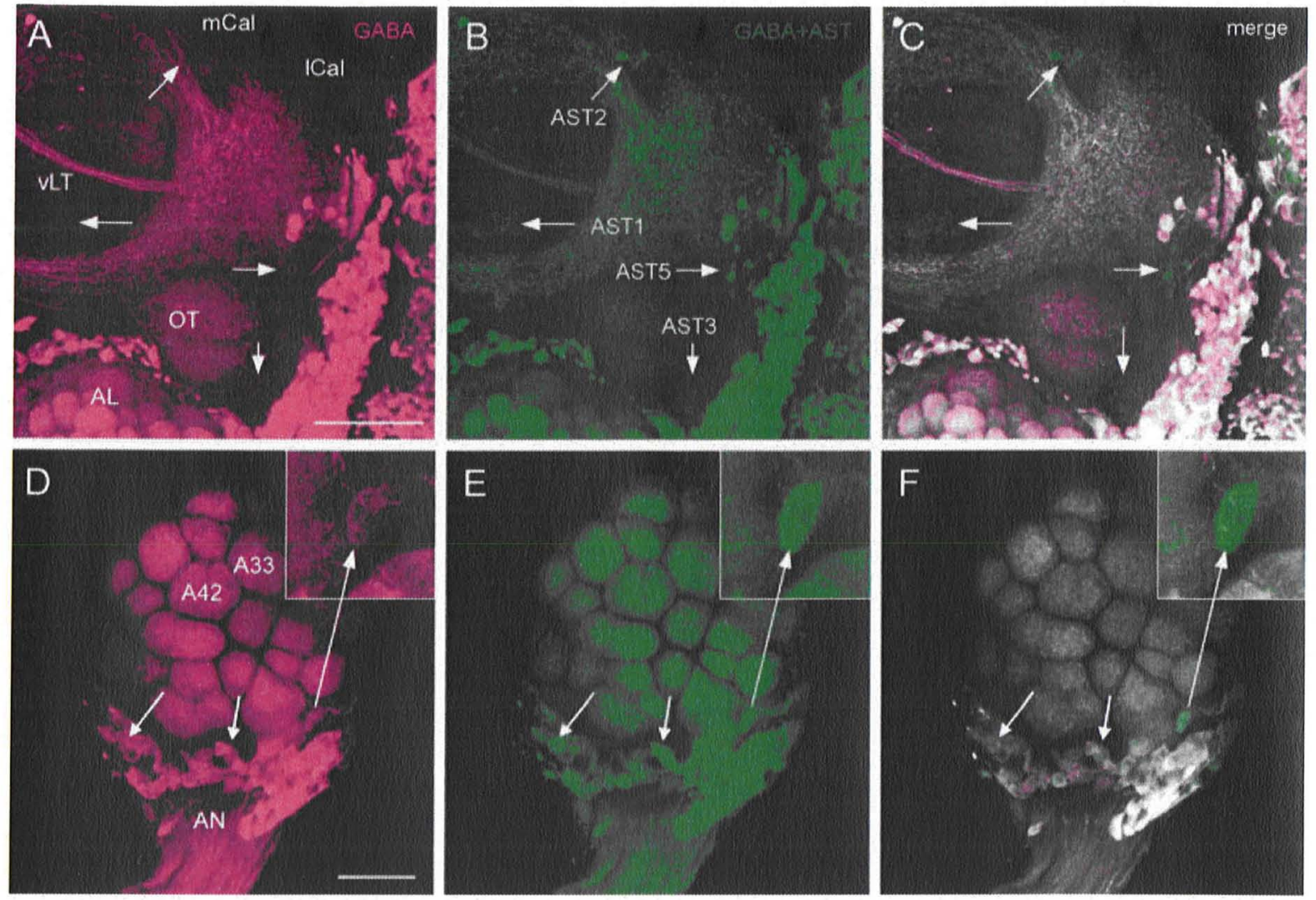

Figure 10. Double immunolabeling of GABA and AST in the brain (A-C) and in the AL (D-F). A,D: Staining against GABA shown in magenta. B,E: Subsequent staining of the same preparation against AST and detection of the primary antibodies with another fluorophore label AST and may additionally label GABA. Thus, neurons containing either exclusively AST, GABA, or, both, are shown in green. C,F: In the overlay, exclusively green-labeled neurons indicate that they were only stained with the second fluorophore, thus only contain AST but no GABA. These are neurons in the groups AST1, AST2, AST3, and AST5 (arrows in A-C) and some fibers in the oblique vertical lobe tract. No neurons exclusively displaying the green label were found in the AL, although GABA was occasionally only faintly stained (see insets in D,E). Thus, GABA was colocalized with AST in all ASTir neurons of the antennal lobe. For abbreviations, see list. Scale bars $=100 \mu \mathrm{m}$ in A (applies to B,C); $50 \mu \mathrm{m}$ in D (applies to E,F).

at the anterior surface of the vertical lobe neuropil (Fig. 6A,B, Supporting Movies SM1, SM3, SM4). After passing the vertical lobe the neurites reentered the medial protocerebral neuropil, superficially diving in a mediodorsal direction. They passed by the central trachea and entered the tangled neuropil, where they could not be followed any further. In specimens double-stained for GABA and AST we additionally found GABAir neurites running in the VLT. At least eight of the AST5 somata showed no GABA staining (Fig. 10A-C) and a minimum of seven neurites in the $V L T$ were not GABAir, which together indicates that AST5 neurons did not exhibit colocalized GABA immunostaining.

\section{AST6, AST7}

These neurons formed a population of about 20 cells associated with the AL and mostly located laterally of it
(Figs. 1A,B,E, 2B), with AST6 being close to the antennal nerve and AST7 close to the protocerebrum. Their stained neurites appeared to be limited to the AL neuropil (see below and Figs. 8-10).

\section{AST8}

This group was found in an area close to or within the median neurosecretory cluster. Neurons in this cluster project through the nervus corporis cardiacus I described by (Eichmüller et al., 1991). It contained two big (34-20 $\mu \mathrm{m}$ and 20-27 $\mu \mathrm{m})$ and about eight smaller $(7-16 \mu \mathrm{m})$ somata on each side of the brain midline (Figs. $1 \mathrm{~A}, \mathrm{~B}, \mathrm{E}$, $2 A)$. The big somata lay above the anterior face of the $C B$ (Figs. 4A, 5C). Each had a stout (up to $2.5 \mu \mathrm{m}$ diameter) but faintly stained primary neurite, which projected posteriorly and then turned behind the $\mathrm{CB}$ in a ventral direction. The neurites then followed a straight trajectory 


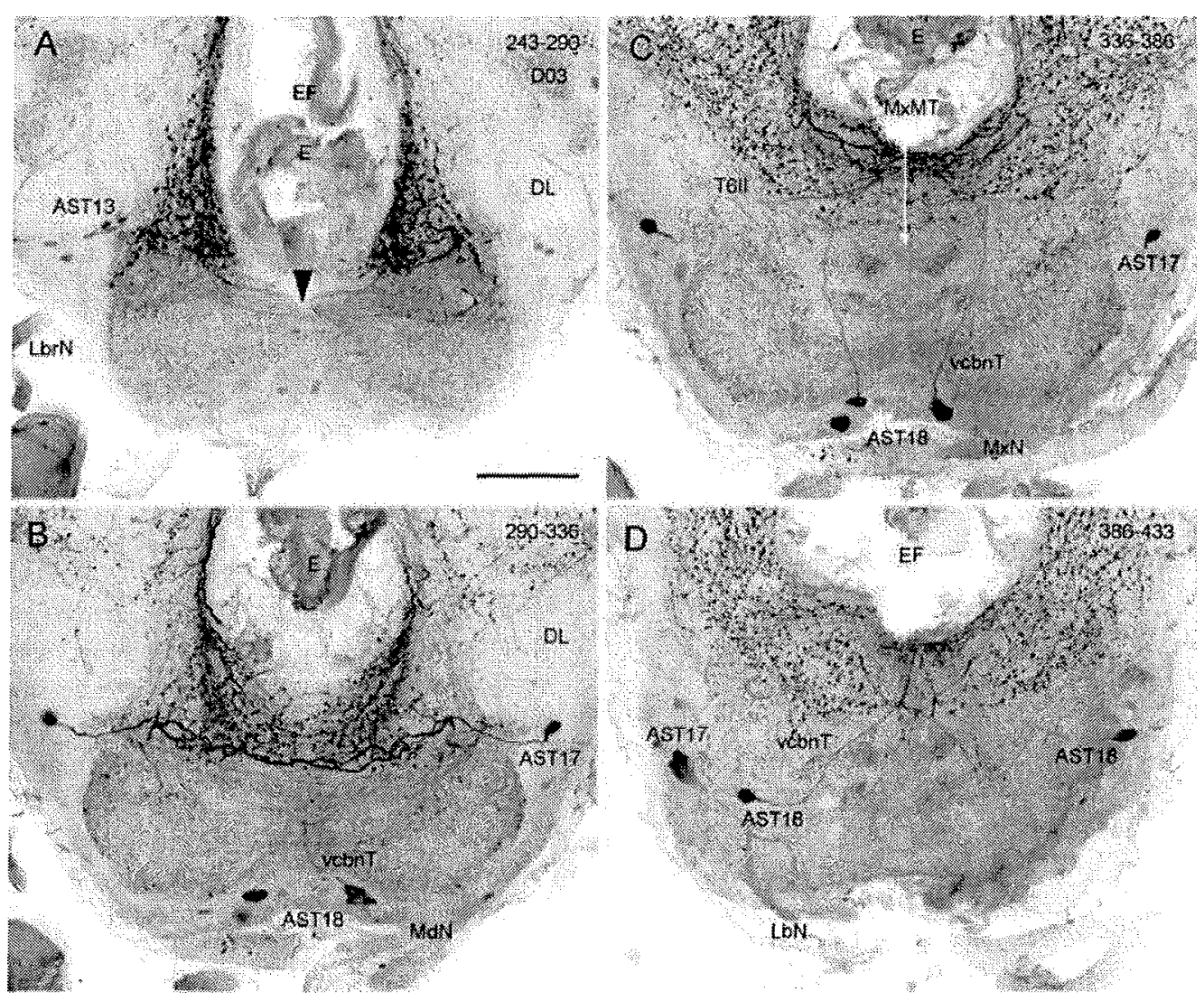

Figure 11. Subesophageal ganglion, consecutive frontal projections with depths indicated in $\mu \mathrm{m}$. About 20 neurites cross the midline close to the esophagus (arrowhead in A). Neurites from the AST17 group (see A,B) contribute to the strong innervation around the esophagus, where they join other ASTir neurites (e.g., from AST 15, see Fig. 5D). Neurites from the AST18 group enter the vcbn tracts of the mandibular, maxillar, and labial neuromeres toward the esophagus $(B, C)$. Three innervation modes can be distinguished in the SEG: dense innervation around the esophagus (A), blebby and diffuse innervation more posteriorly $(C, D)$, and sparkled and diffuse innervation in the remaining areas. For abbreviations, see list. Scale bar $=100 \mu \mathrm{m}$ in A (applies to all).

toward the esophageal foramen without forming visible branches. They then crossed the midline close to the esophagus and here they gave rise to side branches that extended into the inferior protocerebrum, into the posterior deutocerebrum, and into the subesophageal ganglion. They followed the bent neuraxis along the proximal side of the circumesophageal neuropil remaining contralateral. They appeared to enter the cervical connective, suggesting that they may project into the thorax. The small neurons within AST8 were scattered above and behind the $C B$. Their primary neurites could not be traced. This group was separated from group 15 by the ocellar giant interneuron tract.

\section{AST9.}

This strongly stained group (Figs. $1 \mathrm{~A}, \mathrm{~B}, \mathrm{E}, 2 \mathrm{C}$ ) consisted of $\approx 60$ cells with a subgroup with relatively large cell bodies, closely clustered in the anterior soma layer between lateral protocerebrum and the medulla. Their pri- mary neurites projected posteriorly and then bifurcated. One bundle extended into the protocerebral neuropil. The other bundle projected into the distal part of the medulla bypassing the lobula.

\section{AST10}

These $\approx 75$ somata were arranged anteriorly between the lateral protocerebrum and the medulla, forming a vertical arc around the optic tubercle tract (Figs. 1A,C,E, 2C, $3 C-F)$. Their neurites projected posteriorly creating a radiating structure of neurites invading the lobula (Fig. 3D-F). At least some of these neurons traversed the inner chiasma and extended into the proximal part of the medulla, but it remained unclear whether all of them had this morphology.

\section{AST11}

This large group of about 200 small cell bodies (Figs. $1 A, C, E, F, 2 C, 3 D$ ) was located below the proximal 
medulla. Although no neurites could be traced, we assume that these neurons may contribute to the strong network in the medulla.

\section{AST12}

This group of about 18 ASTir somata was located medially of the posterior margin of the AL (Fig. 1A,C,F, 2D), in close vicinity to the esophageal foramen, corresponding to the location of the A4-group of MB extrinsic neurons described previously (Rybak and Menzel, 1993). We made mass fills with tracer-dye from the MBs and combined them with AST immunostaining, confirming that AST12 neurons are indeed scattered within the A4 group (data not shown). However, ASTir neurons in this group did not project into the MBs, but projected into the club-shaped tritocerebral neuropil median of the dorsal lobe, with further projections into the subesophageal ganglion.

\section{AST13}

This group of about 10 somata was located ventral to the dorsal lobe (Figs. 1A,C,F, 2D). The neurites projected toward the tritocerebrum.

\section{AST14}

This group of $9-10$ cells was located laterodorsally to the dorsal lobe (Figs. 1A,C,F, 2D). The group may in fact be continuous with AST 16. We separated the two clusters at the posterior margin of the dorsal lobe.

\section{AST15}

A large group of about 73 cells with heterogeneous morphology (Figs. 1A,D,F, 2E, 5A,B) was found behind the $C B$ in the soma layer of the posterior superior protocerebrum, also known as the posterior pars intercerebralis. Most somata with small diameters $(6 \mu \mathrm{m})$ had only barely detectable neurites, apparently projecting into the central complex. Three prominently large $(16-22 \mu \mathrm{m})$ and strongly stained somata in this group (15A, 15B, 15C), however, had a distinct projection pattern. AST15A and AST15B formed a pair of cells posterior of the protocerebral bridge. They were located medially behind the medial pedunculus, or between the medial and lateral pedunculus (Fig. 5B,C). These neurons did not exhibit GABA and AST colocalization (data not shown). Their neurites dived anteriorly under the protocerebral bridge and continued upward in front of the protocerebral bridge, where they joined the lateral rim of the median antennocerebral tract (mACT) for a short distance. The thick and clearly stained primary neurites then left the mACT at its entry to the pedunculi and continued their anterolateral trajectory above the dorsomedial rim of the vertical lobe. Finally, they turned ventrally along the median border of the verti- cal lobe and supplied the inferior median protocerebrum with dense and varicose ASTir arborizations. It remained unclear whether these neurons may also project contralaterally near the horizontal AST 1 tract.

AST 15C lay more laterally than the other two big AST15 neurons, behind the lateral pedunculus (Fig. 5D). The primary neurite projected anteriorly and bifurcated between the medial and the lateral pedunculus into an anterior and a posterior branch (Fig. $5 \mathrm{C}$ ). The posterior branch followed a sharp U-turn between the pedunculi, turned ventrally, and formed several side branches that contributed to the AST supply into the inferior and the lateral protocerebrum. The anterior branch continued medially and wrapped around the medial pedunculus, turned slightly dorsally to cross the path of the AST15A and AST15B neurites. Along the path, it formed several side branches supplying the anterior protocerebrum. The main branch continued ventrally, crossed the midline (Fig. 5E), and projected along the esophageal foramen into the SEG.

In addition the AST $15 \mathrm{C}$ neurons had a projection passing $30 \mu \mathrm{m}$ anterior of the medial tip of the medial lobe and moving ventrally toward the esophageal foramen. It projected further into the subesophageal ganglion, from where it passed through the subesophageal ganglion into the cervical connective.

\section{AST16}

This group consisted of $8-10$ small (diameter $\approx 5.5$ $\mu \mathrm{m}$ ) and 4 medium-sized (diameter $\approx 10 \mu \mathrm{m}$ ) somata located medially to the lobula (Figs. IA,D,F, 2E). Their neurites projected anterodorsally. The larger neurites gave rise to sparse projections into the superficial lateral horn (LH) neuropil. The smaller neurites continued anteriorly into the inferior anterior protocerebrum. In double immunostainings we found two somata that were clearly ASTir and GABA-negative.

\section{AST17}

This group was located in the lateral soma rind of the subesophageal ganglion and consisted of about nine heterogeneously stained somata (Figs. 1A,D,F, 2F). The group contained a single, prominently stained larger neuron, which projected medially and anteriorly, trifurcated, and supplied a net of neurites in the median tritocerebral neurohemal surface at the esophageal foramen (Fig. $11 \mathrm{~A}-\mathrm{C})$. A good part of the neurons in this group had no discernable neurites, making them orphans in our preparations.

\section{AST18}

These somata were located in the ventral soma rind of the subesophageal ganglion (Figs. 1A,D,F, 2F, 11A-D) 
and consisted of seven neurons in each side of the ventral cell body neuron group (Rehder, 1988). The initial segment of the neurites was traceable in some, but not all of these neurons and projected into the ventral cell body neuron tract (Fig. 11C,D).

\section{Optic lobes}

The optic lobes consist of lamina. (distal), outer chiasma, medulla, inner chiasma, and lobula (proximal). The neuropil of the lamina has been divided into layers $A$ (distal) to C (proximal) (Ribi, 1974). The medulla can be subdivided into strata 1 (distal) to 6 (proximal) and the lobula can be divided into strata 1 (distal) to 4 (proximal) (Ribi and Scheel, 1981). ASTir was strongly stratified in the optic lobes (Figs. 1B, 3A-l; see also Supporting Movie SM2).

In the most distal optic neuropil, the lamina, layer C was densely supplied by ASTir fibers. The origin of these fibers could not be clearly attributed to a soma group. They could be traced from the vicinity of AST9 AST10 somata. They bypassed the anterior surface of the medulla, and then projected radially through the dorsal region of the outer chiasma to the posterior rim of the lamina. Here they entered the lamina, most of them in loose bundles of few neurites, and established a fine tangential net in all parts of the lamina C-layer (Fig. IF, Supporting Movie SM2).

In the medulla the distal layers 1,2, and 3, were supplied by AST 9 neurites and the more proximal layer 5 was densely supplied by AST10 neurites. Neurites of AST9 neurons bypassed the anterior surface of the lobula by forming a fan of horizontally and ventrally extending fibers. These fibers formed many centripetal side branches, which entered the anterior rim of layer 3 of the medulla. They sent off a dense plexus of varicose tangential branches, which contributed to the innervation throughout layer 3 and layer 2 and extended many centripetal blebby neurites into medulla layer 1 (Fig. 3D-1, Supporting Movie SM2).

AST10 primary neurites projected posteriorly, entered the lobula through the anterior fiber pathway, and spread to a posterior-ventrally opened fan into the lobula. They traversed strata 6 and 5 and further radiated within stratum 4 of the lobula neuropil, without apparent ramifications. They branched extensively in the distal lobula strata 1 to 3 with the densest plexus formed in the superficial layer of stratum 1 (Fig. 3F-1). At least a subpopulation of AST10 neurites passed through the inner chiasma and entered the medulia. They traversed the proximal layer 6 of the medulla and finally formed a dense plexus of fine fibers in layer 5 (Fig. 3F-I, Supporting Movie SM2). AST11 neurons and neurites from the protocerebrum likely contribute at least to the ASTir innervation of stratum 5 .

\section{Central complex}

The central complex consists of the protocerebral bridge $(\mathrm{PB})$, the upper division (fan-shaped body, with very few GABAir fibers), the lower division (ellipsoid body, with a very dense GABAir innervation), and the noduli. PB and noduli contain no GABAir processes (Homberg, 1985; Schäfer and Bicker, 1986). All of these were invaded by ASTir neurites (Fig. 4). Staining in the upper and in the lower division was radially patterned, following the radially segmented structure of this neuropil. At least part of this staining likely originated from the small somata of group AST15, which also contributed to the fine staining in the protocerebral bridge. After leaving the protocerebral bridge anteriorly, some neurites entered the upper $\mathrm{di}$ vision of the $\mathrm{CB}$ on the ipsilateral side, whereas others crossed the midline to supply the contralateral hemisphere of the CB (Figs. 4B, 5C,D). The fibers appeared to continue to the lower division. However, the lower division exhibited slightly less densely packed ASTir neurites as compared to the upper division (Figs. 4, 5C,D).

A group of fibers left the $C B$ through the isthmus tracts on both sides and appeared to terminate with diffuse large-diameter terminals above the ALs in the lateral and ventral regions of the protocerebral hemispheres (Fig. $5 E$,F, Supporting Movie SM1) also referred to as the lateral accessory lobes (Homberg, 1985).

\section{Protocerebral neuropil (without MBs)}

Dense ASTir innervation was apparent throughout most regions of the protocerebrum and particularly prominent in the frontal ring neuropil around the vertical lobes (Figs. 1E, 2A,C, 6). The bulk of the network consisted of varicose neurites with variable diameters of 0.2 to about $1 \mu \mathrm{m}$. They were filled with granular staining, with granules about $0.2 \mu \mathrm{m}$ in size that were packed inhomogeneously and often gave a discontinuous appearance to the neurites. Many neurites were decorated with multiheaded boutons of about $1.5 \mu \mathrm{m}$. In the ring neuropil there were large neurites of $0.7 \mu \mathrm{m}$ diameter with a straight and smooth appearance that we traced to AST15A,B,C (Figs. $6 B-F, 7)$.

The area between the two vertical lobes consisted of a symmetric meshwork spanning across the brain midline (Figs. 1E, 2B).

The lateral protocerebral lobe and the $\mathrm{LH}$ are target areas of $\mathrm{MB}$ extrinsic neurons that come from the vertical lobe (Rybak and Menzel, 1993; Strausfeld, 2002), and for olfactory projection neurons from the AL, including the multiglomerular ml-ACT neurons, which form a "triangle" in the lateral network of the protocerebrum (Kirschner 
et al., 2006). Neurites from the AST3 group invaded this triangle (Supporting Movies SM1, SM3). Olfactory PNs travel in the ACT tracts across the protocerebrum. Thorough investigation of specimen, in which the median and the lateral ACTs were stained by mass fills, revealed that these prominent tracts were devoid of AST immunostaining (data not shown but compare also Figs. 4B-D, 5B). We conclude that uniglomerular PNs do not express AST.

The $\mathrm{LH}$ was innervated by fine neurites $(0.3 \mu \mathrm{m}$ diameter with fine varicosities of $0.6 \mu \mathrm{m}$ ) in a very sparse manner at depths of $80-235 \mu \mathrm{m}$ from the frontal surface (Fig. $3 \mathrm{~B}-\mathrm{D})$. At least one neurite supplying the $\mathrm{LH}$ could be traced to the posterior protocerebrum at $350 \mu \mathrm{m}$ depth from the frontal surface and appeared to originate from AST16 somata.

The bulk of neurites in the inferior posterior protocerebrum had a distinct morphology. They formed short side branches and multiheaded terminal boutons which contacted sensory projections from the AN in the dorsal lobe (data not shown). These neurites were $0.25 \mu \mathrm{m}$ in diameter and were decorated with varicosities up to $1.5 \mu \mathrm{m}$ in diameter. The varicosities were irregularly spaced, with interbouton distances in the range of 3-10 $\mu \mathrm{m}$ (Supporting Movies SM3, SM8). The corpora cardiaca of the neurohemal retrocerebral complex exhibited dense ASTir innervation (Supporting Fig. SF1)). It could not, however, be traced to any cellular origin.

\section{Mushroom bodies}

In honeybees the MBs consist of two calices that receive input from optical and olfactory sensory areas. Their intrinsic cells are the Kenyon cells (KCs), which have their somata along the lips and within the cups of the calyces. $\mathrm{KC}$ axons travel along the pedunculi, where most of them branch to form the medial lobe with one branch (corresponding to the Drosophila horizontal lobe), while the other branch contributes to the three dorsal divisions of the vertical lobe. The ventral division of the vertical lobe, also termed the $\gamma$-lobe, is formed by a specific subpopulation of $\mathrm{KCs}$, the so-called clawed $\mathrm{KCs}$ (Strausfeld, 2002). Feedback neurons from the lobes to the calyx travel in the protocerebral-calycal-tract (PCT), which has been subdivided in the distinct subpopulations $A 3 v$ and $A 3 d$. Other MB extrinsic neurons include the A4 group (Rybak and Menzel, 1993; Grünewald, 1999). We identified the subdivisions of the vertical lobe in ASTstained brains by mass filling the PCT neurons from their entry to the MBs between the calices (data not shown) and nuclear staining of glial cells within the neuropil.

AST staining was highly structured in the MBs, with distinct patterns in both of the lobes, the pedunculi, and in the basal ring and in restricted areas of the calyces (Figs. $5 \mathrm{~B}, 6,7)$. To the best of our knowledge, most if not all
ASTir neurites in the MB lobes and calyces derived from the AST3 group. The AST3 group lies in the soma layer beneath the optic tubercle at a depth of $30-80 \mu \mathrm{m}$ from the anterior surface of the brain (Figs. 1, 2B, 3A,B, 8B,C, $10 \mathrm{C}$ ). These neurons did not double stain for GABA (Fig $10 A-C)$. They sent off minute faintly stained primary neurites that dived dorsoposteriorly behind the optic tubercle tract up to the point where the paths of $A 3 v$ and $A 3 d$ PCT neurites converge. From there, the AST 3 neurites followed a more dorsal and more posterior route than the PCT, possibly bypassing the anterior commissure posteriorly, and rejoined the PCT anteriorly between the calyces (Figs. 6A-E, 7A, see also Supporting Fig. SF1 D-F and Supporting Movies SM1, SM3). Here the neurites divided, following two different routes to finally supply the $M B$ lobes. The first group of about 16 collaterals supplied the lobes directly, without entering the calyces. From the bundle of ASTir fibers beneath the frontal part of the calyces they dived ventrally into the pedunculi, which they transversed and then extensively contributed to the meshwork of ASTir neurites in the medial and vertical lobes (Fig. 6E, Supporting Movies SM1, SM3). The second group of branches, however, took a more tortuous route to the lobes. First, they climbed dorsally into the necks of the calyces, where they also sent side branches into parts of the calyces (Fig. 6E, Supporting Movies SM1, SM3). These neurites ran through the inner ring tract and sent off dorsal branches, which climbed to the dorsal rim of the basal ring. There they sent off a few projections toward the ventral rim of the lip along the bundles formed by $\mathrm{KC}$ axons. These bundles were about $15 \mu \mathrm{m}$ apart. Other branches surrounded the dorsal rim of the basal ring radially and sent projections into the outer basal ring, which receives projections of visual interneurons from the optic lobes (Gronenberg, 2001). Both in the ventral lip and the outer basal ring the neurites were faint and ended blindly by forming small blebs of $\approx 1 \mu \mathrm{m}$ diameter (Figs. $6 \mathrm{C}, \mathrm{D}, 7 \mathrm{~A})$. A mesh-like tube of minute obliquely interwoven neurites extended from the inner rim of the inner ring tract down into the pedunculi. From AST-immunostained specimens in which either PCT or ACT neurons were labeled by mass fills, it was clear that these ASTir fibers ran centrally to the PCT and ACT neurites (data not shown). The tube opened posteriorly to prepare for the transformation of the radial calycal geometry into the linear lobe geometry. About 16 very fine and varicose neurites extended from this inner tube-like mesh and projected ventrally through the pedunculi toward the lobes (Fig. $6 \mathrm{D}, \mathrm{E})$. They branched extensively before supplying the lobes where they formed distinct bands of ASTir innervation. A $25-\mu \mathrm{m}$-thick layer of ASTir neurites was prominent in the ventral division of the vertical lobe (Fig. 6A-C, Supporting Fig. SF1G-I, Supporting Movie SM4). This band 
corresponded to layer 4 in Mobb's description (Mobbs, 1982a; Strausfeld, 2002) and may overlap with the layer that is supplied by the A4 vertical lobe extrinsic neurons described previously (Rybak and Menzel, 1993). The network of stained neurites in the vertical lobe continuously extended from its anterior surface throughout the horizontal straight part to the pedunculi (Fig. 6A-E, Supporting Movie SM3). The neurites formed a loose mesh of horizontally and vertically traveling segments. In the frontal part of the vertical lobe we found more trajectories extending laterally in the horizontal plane. In more posterior parts, anterior-posterior and vertical trajectories were predominant. The ASTir neurites in the vertical lobe were not double-labeled for GABA and ASTir and GABAir bands were clearly separated in the basal ring of the calyces (data not shown). The strata of the $\gamma$-lobes, also contained ASTir neurites but were less densely supplied. We found no larger neurites, and no neurites entering the $\gamma$ lobes directly from the protocerebrum.

A vertical mesh of fine neurites filled a layer of the anterior $30 \mu \mathrm{m}$ in the medial lobes. These varicose neurites were up to $0.5 \mu \mathrm{m}$ in diameter. The posterior $70 \mu \mathrm{m}$ of the medial lobes were devoid of ASTir neurites. However, it was not possible to decide from our scans which ASTir neurites in the lobes derived from the direct path to the lobes and which derived from neurites taking the tortuous route through the calyces.

\section{Antennal lobe}

The honeybee ALs are innervated by four tracts of the antennal nerve. $T 1, T 3$ supply $\approx 70$ glomeruli each, and $\mathrm{T} 2$, T4 supply $\approx 7$ giomeruli each. Receptor neurons from the antenna.innervate predominantly the outer cap of each glomerulus for T1-T3, but the entire glomerulus in T4 (Pareto, 1972; Arnold et al., 1985; Galizia et al., 1999; Nishino et al., 2009). Uniglomerular PNs leave the ALs via two tracts. The IACT contains axons of PNs from all glomeruli that are innervated by T1 (A glomeruli) and from some glomeruli that are innervated by $\mathrm{T} 2$ or $\mathrm{T} 3$ ( $\mathrm{B}$ or $\mathrm{C}$ glomeruli, respectively). The mACT contains axons from all glomeruli that are innervated by $\mathrm{T} 3$ and from some glomeruli that are innervated by $\mathrm{T} 2$ or $\mathrm{T} 4 \mathrm{~B}$ or $\mathrm{D}$ glomeruli, respectively) (Bicker et al., 1993; Abel et al., 2001; Kirschner et al., 2006). About 4,000 LNs branch locally in the ALs (Witthöft, 1967).

We found a total of $\approx 20$ ASTir somata associated with each AL (18.6 $\pm 3.2 \mathrm{SD}, \mathrm{n}=51 \mathrm{ALs})$. Their neurites invaded the central coarse neuropil and from there supplied all glomeruli in a comparable pattern and density, creating a fairly uniform view of the entire AL (Figs. 8, 9). ASTir neurites entered all glomeruli, including glomerulus D08 (Kirschner et al., 2006). No apparent difference was visible between glomeruli associated with the IACT and those associated with the mACT. However, all ASTir neurons appeared to have primary branches leading to T4 glomeruli and other branches leading further into the other glomeruli (Supporting Movies SM5, SM6). We could not reliably determine the number of ASTir neurites invading each glomerulus, but in good scans the number was about five. ASTir neurites innervated T4-glomeruli across the entire volume of the glomeruli, while the staining was concentrated to the central area in the other glomeruli (Fig. 9). Double staining with mass fills of olfactory receptor neurons (ORNs) showed that the area mostly covered by ASTir neurites corresponded to the area not covered by ORNs (Fig. 9A-C), suggesting that the major synaptic contact of ASTir neurons in glomeruli is not with ORNs. However, we observed a prominent overlap of bleb-like swellings of ASTir neurites at the interface between the glomerular cap and the core (Fig. 9D,E). Moreover, a few ASTir fibers entered the cap, leaving the possibility of contacts between ASTir neurons and ORNs

A nonglomerular projection area of the antennal nerve afferents in the posterior central neuropil of the AL was completely devoid of ASTir neurites (Supporting Movies SM5, SM6). This area was previously termed "wulst" or "worm like region" (Pareto, 1972) and is also referred to as the central tract (Galizia et al., 1999; Kirschner et al., 2006).

The location of the somata contributing to the innervation of the $A L$ was strikingly variable across different animals (compare Figs. 1E, 2B, 8, 10), and even differed between the two ALs of an individual animal. The two soma groups described here (AST6 and AST7) are classified based on the trajectories of their primary neurites entering the AL (Fig. 8). Group AST6 formed a loose necklace around the entrance of $T 1$, extending from the medioventral to the lateral soma cortex of the AL. Most primary neurites of AST6 entered the AL below T1. If, in a given specimen, they were found on both sides of the AN they projected through a small furrow dividing T1 glomeruli from T2, T3, and T4 glomeruli. We also found ASTir somata in a ventral position behind the antennal nerve with neurites trajecting between some T3 glomeruli into the central neuropil of the AL. In some specimens a few somata belonging to AST6 were found to be flipped upward before the most anterior glomeruli, or even in a mediodorsal position of the AL soma cortex. In very few cases the primary neurites of anteriorly flipped somata entered the AL between $\mathrm{T} 1$ glomeruli (Supporting Movie SM7).

The AST7 group was located in a dorsolateral position in the posterior part of the lateral soma cortex of the $\mathrm{AL}$, within the lateral soma cluster and the lateral part of the medial soma cluster of PNs as described by Kirschner et al. (2006). Their primary neurites projected 
ventroanteriorly and entered the $\mathrm{AL}$ either through the most posterior part of the lateral passage or behind T4-2 and T4-3.

No staining was found in the antennal nerves, suggesting that no sensory neurons were ASTir. Similarly, no neurites appeared to leave the AL, suggesting that all ASTir neurons were LNs. At the AL exit of the ACTs a loose bundle of about five fine $(<1 \mu \mathrm{m})$ ASTir neurites that derived from the dorsal lobes joined the mACT (Fig. 2D). This bundle followed the path of the mACT up to a point beneath the medial lobe, where it left this tract laterally. On the route of the $\mathrm{MACT}$ along lateral flanks of the $\mathrm{CB}$ we only found ASTir neurites, which clearly could be attributed to AST15. We never found ASTir neurites joining the IACT (Figs. 4B-D, 5B). However, we cannot entirely exclude that there may be minute connections between $A L$ and $\mathrm{DL}$, given that staining in the $\mathrm{DL}$ was very sparse and faint.

All ASTir neurons in the AL showed colocalized GABA immunostaining (Fig. 10D-F). GABA has been suggested to be the dominant transmitter of LNs in the honeybee antennal lobe (Schäfer and Bicker, 1986). Within the population of GABAir cell bodies, AST- and GABA-double-labeled somata were among the larger ones, but there were many other large GABAir cells that were not ASTir, in addition to a very large group of smaller GABAir cells.

\section{Subesophageal ganglion}

Several ASTir tracts and commissures were found in the subesophageal ganglion, varying in dimension from large neurites $(>2.1 \mu \mathrm{m})$ to very small processes $(0.2 \mu \mathrm{m})$. We found about 20 neurites interconnecting the two hemispheres (Fig. 11A,B,). Neurites from the AST17 group entered the tangled neuropil and one particularly prominent neuron formed a dense plexus in the tritocerebral area. Neurites from the AST18 group entered the SEG through the ventral cell body neuron tracts (Fig. 11B-D, Supporting Movie SM8). A few ASTir neurites were found in the nerve roots of the mandibular and the maxillar nerve, and the frontal nerve consistently carried a number of ASTir neurites. We did not attempt to elucidate their further destination (Supporting Movie SM7). About 20 neurites ran through the cervical connective toward the thorax.

\section{DISCUSSION}

\section{Distribution of AST in the bee brain}

Communication within the brain is accomplished by a small family of "classical" neurotransmitters and a large and increasingly well-known family of peptides. Here we describe the distribution of substances related to DipAST I in the honeybee brain. We show that of all neuro- peptides characterized so far, ASTir neurons form the largest network, with neurons contributing to the neuropil in almost all subsystems of the brain, both in terms of cell number, as well as in terms of neuropil density, including FMRF and GCCK (Schürmann and Erber, 1990; Eichmüller et al., 1991; Strausfeld et al., 2000), dopamine (Schäfer and Rehder, 1989; Kirchhof et al., 1999), octopamine (Kreissl et al., 1994; Sinakevitch et al., 2005), and histamine (Bornhauser and Meyer, 1997). The only other similarly widespread labeling for either peptides or amines that we know of is with the biogenic amine serotonin (Schümann and Klemm, 1984; Rehder et al., 1987).

ASTir neurites are found in the optic lobes, medulla, lobula, and lamina. They are found in all areas associated with olfaction, the ALs, the MBs, and the lateral horn, as well as in the CB and premotor areas. In several instances, ASTir neurons form distinctly local networks, e.g., within the AL, within the MBs, and within the optic lobes. Many of the neurons labeled in these networks have a morphology of "small neurons" (Nässel et al., 2008) and are likely to form point-to-point synaptic contacts with other neurons.

In addition, there are large ASTir neurons with the morphology of neurosecretory cells that are likely not pointto-point in their AST release. These are median neurosecretory cells and neurons in the posterior pars intercerebralis (e.g., the neurons AST 8 and AST 15A, B, and C; see Fig. 6). Moreover, several neurites were found connecting the brain with the thoracic ganglia. The lateral neurosecretory cells of the honeybee contributing to the innervation of the retrocerebral complex are distributed anterior to the peduncle of the lateral calyx (Eichmüller et al., 1991). In contrast, AST4 neurons are scattered around the median calyx. The distributions of AST4 somata and of the lateral neurosecretory cells may only marginally overlap between the median and the lateral calyces. AST5, AST9, and AST 10 were located in more lateral and posterior positions (Figs. 1, 2A,C). However, AST is likely to play a role as a neurohormone in the honeybee's hemolymph, because we found ASTir fibers in the corpora cardiaca. Unfortunately, we could not trace these neurites back to their cell bodies. It thus remains open whether some of the AST4 neurons are neurosecretory cells contributing to the staining in the corpora cardiaca. Neurohemal structures that stain for AST have also been found in other insects (Stay et al., 1992; Stay et al., 1994; Veelaert et al., 1995b; Maestro et al., 1998; Stay and Tobe, 2007) but the functional role of AST-A peptides is not uniform across this phylum: in cockroaches and crickets they consistently act as inhibitors of juvenile hormone synthesis, while no such effect could be shown in locusts, flies, and bees (Veelaert et al., 1995b; Rachinsky and Feldlaufer, 2000; Stay and Tobe, 2007). 


\section{Physiological action of AST}

AST acts as an inhibitor in visceral muscles of many species (Duve and Thorpe, 1994; Duve et al., 1995; Veelaert et al., 1996). In the honeybee brain we also assume that AST is inhibitory. Evidence for this proposal is the widespread colocalization of AST and the inhibitory neurotransmitter GABA. Neurons that colocalize GABA and AST are also known from locusts and moths (Vitzthum et al., 1996; Berg et al., 2009). In the stomatogastric systems of two astacuran species, AST leads to increased spiking precision, mediated by its inhibitory action (Billimoria et al., 2006). Our finding that ASTir networks are local to their own area (e.g., within the ALs) would make a function related to spike-time precision plausible, but the number of large neurons in other brain regions would suggest that this is not the only function of AST. Furthermore, there are important subpopulations of ASTir neurons that are not GABAir (Fig. 10). For example, we could not find any evidence for GABA colocalization in the AST3 group within the MBs (Fig. 10A-C). These neurons may only use AST, or they may coexpress other neurotransmitters.

\section{ASTir neurons are a diverse family}

Taken together, our observations may suggest that ASTir neurons perform a variety of different roles in the honeybee nervous system. Three of the AST-A peptide isoforms in the honeybee share a posttyrosyl serine with Dip-AST III and Dip-AST IV (Weinstock et al., 2006; Audsley and Weaver, 2006; Hummon et al., 2006; Predel and Neupert, 2007). They are all likely labeled with our antibody, which recognizes peptides with the common -YXFGL-NH $\mathrm{N}_{2}$ signature, including Dip-AST III and Dip-AST IV (Vitzthum et al., 1996). Thus, we may speculate that the diversity of functions suggested by morphology and differences in GABA colocalization correspond to different isoforms. Alternatively, differences might be generated by local network properties, using a common peptide. More selective antibodies are needed to address this aspect.

Members of the AST-A family are common peptides that have been localized in many insects, including Drosophila (Yoon and Stay, 1995), Calliphora (Duve and Thorpe, 1994), Schistocerca (Veelaert et al., 1995a), Locusta (Vitzthum et al,, 1996), Manduca (Davis et al., 1997; Utz and Schachtner, 2005), and Blattella (Maestro et al., 1998). Common to all of these is the frequent but not exclusive colocalization with GABA.

Although the overall distribution of AST immunoreactivity is similar in some insects, the details of ASTir networks differ greatly across species, suggesting that peptidecomplements of neurons were modified in evolution. A distribution of ASTir neurites resembling that found in bees was described in the central complex of locusts. Among others, neurons with their somata in the posterior protocerebrum supply the protocerebral bridge and form columns in the upper part of the central complex (Vitzthum et al., 1996). ASTir neurites have also been found in the central complex of the blowfly (Duve and Thorpe, 1994). In contrast to that similarity, differences exist at a. - more detailed cellular level. There are 40 ASTir cells associated with the AL in Manduca sexta, corresponding to about $13 \%$ of the local neuron population (Utz and Schachtner, 2005), while we found only 20 LNs in honeybee, which together with a much greater number of about 4,000 local neurons in this system (Galizia, 2008) accounts for a mere $0.5 \%$ of this population. Only two pairs of small ASTir neurons project into the antennal lobe in the blowfly (Duve and Thorpe, 1994). Moreover, ASTir local interneurons may not contribute to the antennal network in the cockroach Blatella germanica, although a small number of ASTir tritocerebral neurons connect the tritoterebral neuropil with the periphery of individual glomeruli (Maestro et al., 1998).

\section{Role of AST in the visual system}

In the visual system, ASTir neurites are localized in the lobula and the medulla. Centrifugal ASTir fibers travel from the medulla into the lamina. Here, ASTir neurites are limited to the c-layer, where they might receive direct input from photoreceptor cells.

In a comparison with other insect species we find differences in the details of the ASTir networks in the optic lobes: in the lamina of Schistocerca gregaria ASTir is confined to dorsal and ventral rim areas, while the lamina of the cockroach Leucophaea maderae shows no ASTir (Petri et al., 1995; Vitzthum et al., 1996). In the cockroach, neurons immunoreactive to antibodies against pigment dispersing factor resemble the honeybee ASTir-positive neurons shown here. Furthermore, while in L. maderae and $S$. gregaria ASTir neurites have been found in the accessory medulla, a circadian pacemaker center in the optic neuropils of these insects (Petri et al., 1995; Vitzthum et al., 1996), we found no ASTir neuropil in the corresponding honeybee area.

Neurons with somata in the vicinity of AST9 and AST 10 have been described before in the honeybee (Hertel and Maronde, 1987; Hertel et al., 1987). Those neurons, however, arborized within the intermediate layer 4 of the lobula, and did not project through the inner chiasma to the medulla. They may correspond to a subpopulation of AST10 described here. Serpentine neurons have also been described in this location, but are clearly distinct from the neurons described here because serpentine neurons run in front of the CB horizontally, unlike AST 10 or AST9. Other neurons described in this location were 
immunoreactive to an antiserum against crustacean pigment dispersing hormone, likely to play a role in circadian rhythmicity (Bloch et al., 2003). A possible colocalization of pigment dispersing hormone and AST and/or a role for AST9 and AST10 for circadian rhythmicity in the honeybee remains to be investigated in double labeling and physiological studies.

The tangential morphology of ASTir neurons in the optic neuropils might indicate an involvement with a motion detection network similar to that described in flies (Kalb et al., 2008; Borst, 2009). Motion detection relies on precise spike timing. Given that AST influences precision of spike timing in other systems (Billimoria et al., 2006), we speculate that AST might fulfill a similar task in these neuropils, a proposal that needs to be tested physiologically.

\section{Role of AST in the MBs}

ASTir neurons in the MBs have a morphology that is distantly reminiscent of that of PCT neurons. These neurons branch in the olfactory area of the vertical lobe and the $\gamma$-lobe and have a neurite that then travels back outside the $M B$, providing input to the $M B$ calyces, forming a within-MB feedback loop. These neurons were described in the honeybee and have been reported to be GABAergic (Bicker et al., 1985; Mobbs et al., 1985). PCTs have been proposed to create a feedback that allows for synchronized Kenyon cell activity in the locust system, and that may be necessary for spike-time dependent plasticity (Laurent, 1996). Together with the finding that AST increases spike-time precision in other systems (see above), this offers an intriguing putative function for AST in olfactory learning within the MBs.

It should be noted, however, that the ASTir PCT-like neurons that we found do not reveal exactly the same morphology as those described by intracellular cell labeling (Grünewald, 1999; Abel et al., 2001). One possibility for this discrepancy would be that these neurons form a population of atypical PCT neurons lacking the recurrent path through the protocerebrum. Considering the blebby appearance of the innervation in the basal ring and ventral lip region in the calyces and the fine appearance of ASTir neurites in the lobes, one might suggest that they would instead provide a within-MB feedback system, except for their primary neurites. An alternative possibility would be that ASTir PCT neurons express AST in their somata and in their axons, but not in the dendritic area of the cells, where they do not release the peptide. This would render part of the complete morphology of the neurons invisible in immunohistochemical staining. If the latter is the case, we cannot exclude that this may be true for other ASTir cells as well, i.e., that we might be missing some of the input areas of the neurons described in this study. The fact that for some soma groups we could not detect the primary neurites supports this view. The only possibility to show the complete morphology of ASTir neurons will be in the combination of ASTir with singlecell fills. However, a targeted approach is particularly difficult, given that ASTir somata are not densely clustered in clearly defined areas.

\section{Role of AST in the AL}

A group of $\approx 20 \mathrm{LNs}$ innervated each of the $\approx 160$ glomeruli in the AL equally, indicating that AST6 and AST7 neurons innervate both $A L$ hemi-lobes defined by the two PN tracts (Bicker et al., 1993; Kirschner et al., 2006). LNs in the honeybee $A L$ have two distinct morphologies: one type innervates many glomeruli equally (homo-LN) while the other type (hetero-LN) innervates one glomerulus very densely, and several others only sparsely (Fonta et al., 1993; Sun et al., 1993). If there were hetero-LNs among the ASTir neurons, we would expect 20 or fewer glomeruli to be labeled more densely than the remainder, which is not what we found. We therefore conclude that all ASTir LNs are homo-LNs. At the base of each glomerulus we found 4-5 ASTir neurites, putting the upper limit of ASTir neurons innervating each glomerulus to 5 . Because we cannot trace all neurites unequivocally across the coarse neuropil on their paths to the bases of the glomeruli, it may also be that a single neuron branches into 4-5 neurites before entering a glomerulus, and therefore the lower limit is one ASTir neuron per glomerulus. Thus, each ASTir homo-LN may innervate between 8 (160 glomeruli innervated by 20 ASTir neurons) and 40 (each glomerulus innervated by 5 ASTir neurons) glomeruli.

Olfactory glomeruli in honeybees have a concentric organization (Pareto, 1972; Arnold et al., 1985; Fonta et al., 1993; Sun et al., 1993; Galizia et al., 1999; Nishino et al., 2009): the periphery is innervated by olfactory sensory neuron axons (OSNs). PNs innervate the periphery and the central core and different populations of LNs innervate central and peripheral areas. ASTir neurons innervate the central core area, forming a clear boundary exactly coincident with the boundary formed by sensory neurons (Fig. 9D,E). The ASTir neurites form bleb like boutons in that boundary, and in addition few ASTir neurites branch in the peripheral glomerulus layers, making occasional synapses possible between OSNs and ASTir LNs. They may, however, also have synapses with PNs or other AL extrinsic neurons.

Another indication for the role that AST may play in the AL comes from their morphology. However, all primary neurites of the ASTir neurons straightly invaded T4 glomeruli, suggesting that ASTir neurons in the AL are functionally related to whatever is being processed in $\mathrm{T} 4$ glomeruli. At least some of these glomeruli process hygro- 
and thermosensory signals (Nishino et al., 2009). ASTir neurons may relay these signals within the $\mathrm{AL}$.

All ASTir neurons in the AL showed colocalized GABA immunostaining. However, this population forms but a small subgroup of the $\approx 800$ GABAir LNs in the honeybee AL (Schäfer and Bicker, 1986). Some ASTir somata were large compared to most GABAir cell bodies, but not all large GABAir somata were ASTir, suggesting that within the GABAir population there may be more physiologically distinct subpopulations. It is not known whether a group of preprotachykinin-expressing neurons in the AL belongs to the LN or to the PN population (Takeuchi et al., 2004). It remains unclear whether ASTir neurons may coexpress tachykinin-related peptides in addition to GABA.

The localization of ASTir cell bodies in the AL soma clusters were highly variable, and differed also across the left and right ALs of single individuals. This opens challenging questions for cell-fate determination in development: When in a cell lineage is the AST identity determined? Do the somata travel after being determined? Or are ASTir neurons a subpopulation of "AST-competent" neurons that may even change their expression levels during the animal's lifetime, while keeping their overall number of ASTir cells constant? Many questions remain to be answered.

Most important, however, the precise physiological function that AST plays in the honeybee brain remains to be elucidated. We show here that ASTir neurons form distinct populations with diverse morphologies, branching patterns, and GABA-complement, and that in many areas they contribute to a local rather than to a global computational network. It is likely that the physiological properties of AST also differ in different areas, adding to the already intriguingly complex properties of peptidergic systems in the brain.

\section{ACKNOWLEDGMENTS}

We thank H.J. Agricola (Fiedrich Schiller University, Jena, Germany) for providing the AST antibody and inspiring discussions, which essentially initiated the present study. Thanks to H. Dircksen (University of Stockholm, Stockholm, Sweden) for GABA antibodies. We thank Felix Schönenberger for preparing the tool for labeling of movies. We thank B. Rapp and D. Gustav for the bee keeping indoors as well as outdoors at the University of Konstanz, and P. Knoll (Freie Universität Berlin, Berlin, Germany) for invaluable advice on beekeeping for laboratory use.

\section{LITERATURE CITED}

Abel R, Rybak J, Menzel R. 2001. Structure and response patterns of olfactory interneurons in the honeybee, Apis mellifera. J Comp Neurol 437:363-383.
Arnold G, Masson C, Budharugsa S. 1985. Comparative study of the antennal lobes and their afferent pathway in the worker bee and the drone (Apis mellifera). Cell Tissue Res 242:593-605.

Audsley N, Weaver RJ. 2006. Analysis of peptides in the brain and corpora cardiaca-corpora allata of the honey bee, Apis mellifera using MALDI-TOF mass spectrometry. Peptides 27:512-520.

Belles X, Maestro JL, Piulachs MD, Johnsen AH, Duve $H$, Thorpe A. 1994. Allatostatic neuropeptides from the cockroach Blattella germanica (L) (Dictyoptera, Blattellidae) identification, immunolocalization and activity. Regul Pept 53:237-247.

Bendena WG, Donly BC, Tobe SS. 1999. Allatostatins: a growing family of neuropeptides with structural and functional diversity. In: Sandman CA, Strand FL, Beckwith B, Chronwall BM, Flynn FW, Nachman RJ, editors. Neuropeptides: structure and function in biology and behavior. New York: New York Academy of Sciences. p 311-329.

Berg BG, Schachtner J, Utz S, Homberg U. 2007. Distribution of neuropeptides in the primary olfactory center of the heliothine moth Heliothis virescens. Cell Tissue Res 327: 385-398.

Berg BG, Schachtner J, Homberg U. 2009. gamma-Aminobutyric acid immunostaining in the antennal lobe of the moth Heliothis virescens and its colocalization with neuropeptides. Cell Tissue Res 335:593-605.

Bicker G, Schäfer S, Kingan T. 1985. Mushroom body feed back interneurones in the honeybee show GABA-like immunoreactivity. Brain Res 360:394-397.

Bicker G, Kreissi S, Hofbauer A. 1993. Monoclonal antibody labels olfactory and visual pathways in Drosophila and Apis brains. J Comp Neurol 335:413-424.

Billimoria CP, DiCaprio RA, Birmingham JT, Abbott LF, Marder E. 2006. Neuromodulation of spike-timing precision in sensory neurons. I Neurosci 26:5910-5919.

Bloch G, Solomon SM, Robinson GE, Fahrbach SE. 2003. Patterns of PERIOD and pigment-dispersing hormone immunoreactivity in the brain of the European honeybee (Apis mellifera): age- and time-related plasticity. J Comp Neurol 464:269-284.

Böhm A, Neupert S, Kahnt J, Hedderich R, Predel R, Schachtner J. 2009. A mass spectrometric approach to determine neuropeptides from defined brain regions of Apis mellifera. 8th German Neurosci Conf, Göttingen. p T19-17B.

Bornhauser BC, Meyer EP. 1997. Histamine-like immunoreactivity in the visual system and brain of an orthopteran and a hymenopteran insect. Cell Tissue Res 287:211-22.1.

Borst A. 2009. Drosophila's view on insect vision. Curr Biol 19:R36-R47.

Brockmann A, Annangudi SP, Richmond TA, Ament SA, Xie F, Southey BR, Rodriguez-Zas SR, Robinson GE, Sweedler JV. 2009. Quantitative peptidomics reveal brain peptide signatures of behavior. Proc Natl Acad Sci U S A 106: 2383-2388.

Claeys I, Poels J, Simonet G, Franssens V, Van Loy T, Van Hiel MB, Breugelmans B, Broeck JV. 2005. Insect neuropeptide and peptide hormone receptors: current knowledge and future directions. Vitam Horm 73:217-282.

Davis NT, Veenstra JA, Feyereisen R, Hildebrand JG. 1997. Allatostatin-like-immunoreactive neurons of the tobacco hornworm, Manduca sexta, and isolation and identification of a new neuropeptide related to cockroach allatostatins. J Comp Neurol 385:265-284.

Dircksen H, Skiebe P, Abel B, Agricola H, Buchner K, Muren JE, Nässel DR. 1999. Structure, distribution, and biological activity of novel members of the allatostatin family in the crayfish Orconectes limosus. Peptides 20:695-712. 
Duve H, Thorpe A. 1994. Distribution and functional-significance of Leu-callatostatins in the blowfly Calliphora-vomitoria. Cell Tissue Res 276:367-379.

Duve $H$, Johnsen AH, Scott AG, Yu CG, Yagi KJ, Tobe SS, Thorpe A. 1993. Callatostatins - neuropeptides from the blowfly Calliphora-vomitoria with sequence homology to cockroach allatostatins. Proc Natl Acad Sci U S A 90: 2456-2460.

Duve $H$, Wren $P$, Thorpe A. 1995. Innervation of the foregut of the cockroach Leucophaea-maderae and inhibition of spontaneous contractile activity by callatostatin neuropeptides. Physiol Entomol 20:33-44.

Duve $H$, Johnsen AH, Maestro JL, Scott AG, Crook N, Winstanley D, Thorpe A. 1997. Identification, tissue localisation and physiological effect in vitro of a neuroendocrine peptide identical to a dipteran Leu-callatostatin in the codling moth Cydia pomonella (Tortricidae: Lepidoptera). Cell Tissue Res 289:73-83.

Eichmüller S, Hammer M, Schäfer S. 1991. Neurosecretory cells in the honeybee brain and suboesophageal ganglion show FMRFamide-like immunoreactivity. I Comp Neurol 312:164-174.

Falkenburger BH, Barstow KL, Mintz IM. 2001. Dendrodendritic inhibition through reversal of dopamine transport. Science 293:2465-2470.

Fonta C, Sun XJ, Masson C. 1993. Morphology and spatial-distribution of bee antennal lobe interneurons responsive to odors. Chem Senses 18:101-119.

Galizia CG. 2008. Insect olfaction. In: Basbaum AlK, Shepherd GM, Westheimer $G$, editors. The senses: a comprehensive reference. San Diego: Academic Press. p 725-770.

Galizia CG, Menzel R. 2001. The role of glomeruli in the neural representation of odours: results from optical recording studies. J Insect Physiol 47:115-130.

Galizia CG, Mcllwrath SL, Menzel R. 1999. A digital threedimensional atlas of the honeybee antennal lobe based on optical sections acquired by confocal microscopy. Cell Tissue Res 295:383-394.

Gräbner M, Groh C, Rössler W, Schachtner W. 2005. Development of allatostatin-A in antennal lobe neurons of the honeybee. In: Elsner $\mathrm{H}$, editor. 6th German Neurosci Soc Conf, Göttingen: Thieme. p 832.

Gronenberg W. 2001. Subdivisions of hymenopteran mush room body calyces by their afferent supply. I Comp Neurol 435:474-489.

Grünewald B. 1999. Morphology of feedback neurons in the mushroom body of the honeybee, Apis mellifera. J Comp Neurol 404:114-126.

Hauser F, Cazzamali G, Williamson M, Blenau W, Grimmelikhuijzen C.P. 2006. A review of neurohormone GPCRs present in the fruitfly Drosophila melanogaster and the honey bee Apis mellifera. Prog Neurobiol 80:1-19.

Hertel H, Maronde U. 1987. The physiology and morphology of centrally projecting visual interneurons in the honeybee brain. J Exp Biol 133:301-315.

Hertel H, Schäfer S, Maronde U. 1987. The physiology and morphology of visual commissures in the honeybee brain. Exp Biol 133:283-300.

Homberg U. 1985. Interneurones of the central complex in the bee brain (Apis mellifera, L.). J Insect Physiol 31: $251-261,263-264$.

Homberg U, Vitzthum H, Muller M, Binkle U. 1999. Immunocytochemistry of GABA in the central complex of the locust Schistocerca gregaria: identification of immunoreactive neurons and colocalization with neuropeptides. I Comp Neurol 409:495-507.

Hummon $A B$, Richmond TA, Verleyen $P$, Baggerman $G$, Huybrechts J, Ewing MA, Vierstraete E, Rodriguez-Zas SL,
Schoofs L, Robinson GE, Sweedier IV. 2006. From the genome to the proteome: uncovering peptides in the Apis brain. Science 314:647-649.

Kalb J, Egelhaaf M, Kurtz R. 2008. Adaptation of velocity encoding in synaptically coupled neurons in the fly visual system. I Neurosci 28:9183-9193.

Katz PS. 1999. What are we talking about? Neuromodulation and its importance for information processing. In: Katz PS editor. Beyond neurotransmission. Oxford: Oxford University Press.

Kirchhof BS, Homberg U, Mercer AR. 1999. Development of dopamine-immunoreactive neurons associated with the antennal lobes of the honey bee, Apis mellifera. I Comp Neurol 411:643-653.

Kirschner S, Kleineidam CJ, Zube C, Rybak J, Grünewald B, Rössler W. 2006. Dual olfactory pathway in the honeybee, Apis mellifera. J Comp Neurol 499:933-952.

Kreissl S, Eichmüller S, Bicker G, Rapus J, Eckert M. 1994. Octopamine-fike immunoreactivity in the brain and subesophageal ganglion of the honeybee. J Comp Neurol 348: 583-595.

Kreissl S, Weiss T, Djokaj S, Balezina O, Rathmayer W. 1999. Allatostatin modulates skeletal muscle performance in crustaceans through pre- and postsynaptic effects. Eur Neurosci 11:2519-2530.

Laurent G. 1996. Dynamic representation of odors by oscillating and evolving neural assemblies. Trends Neurosci 19: 489-496.

Maestro J, Belles X, Piulachs MD, Thorpe A, Duve H. 1998. Localization of allatostatin-immunoreactive material in the central nervous system, stomatogastric nervous system, and gut of the cockroach Blattella germanica. Arch Insect Biochem Physiol 37:269-282.

Menzel R, Giurfa M. 2001. Cognitive architecture of a minibrain: the honeybee. Trends Cogn Sci 5:62-71,

Mobbs PG. 1982a. The brain of the honey bee Apis-mellifera 1. The connections and spatial organization of the mushroom bodies. Philos Trans R Soc Lond B Biol Sci 298:309-354.

Mobbs PG. 1982b. The neural architecture of the honeybee mushroom bodies. Proceedings of the International Congress of the International Union for the Study of Social insects 9-369.

Mobbs PG, Kerkut GA, Gilbert LI. 1985. Brain structure. Nervous systems: structure and motor function. Oxford: Pergamon Press. p 299-370.

Nässel DR, Homberg U. 2006. Neuropeptides in interneurons of the insect brain. Cell Tissue Res 326:1-24.

Nässel DR, Enell LE, Santos JG, Wegener C, Johard HAD. 2008. A large population of diverse neurons in the Drosophila central nervous system expresses short neuropeptide $F$, suggesting multiple distributed peptide functions BMC Neurosci 9:90.

Nishino $H$, Nishikawa M, Mizunami M, Yokohari F. 2009. Functional and topographic segregation of glomeruli revealed by local staining of antennal sensory neurons in the honeybee Apis mellifera. I Comp Neurol 515:161-180.

Pareto A. 1972. Die zentrale Verteilung der Fählerafferenzen bei Arbeiterinnen der Honigbiene, Apis mellifera. $Z$ Zellforsch Mikrosk Anat 131:109-140.

Petri B, Stengl M, Wurden S, Homberg U. 1995. Immunocytochemical characterization of the accessory medulla in the cockroach Leucophaea-maderae. Cell Tissue Res 282 3-19.

Predel R, Neupert S. 2007. Social behavior and the evolution of neuropeptide genes: lessons from the honeybee genome. Bioessays 29:416-421.

Rachinsky A, Feldlaufer MF. 2000. Responsiveness of honey bee (Apis mellifera L.) corpora allata to allatoregulatory 
peptides from four insect species. J Insect Physiol 46: $41-46$.

Rehder V. 1988. A neuroanatomical map of the subesophageal and prothoracic ganglia of the honey bee (Apis-mellifera). Proc R Soc Lond Ser B Biol Sci 235:179.

Rehder V, Bicker G, Hammer M. 1987. Serotonin-immunoreactive neurons in the antennal lobes and suboesophageal ganglion of the honeybee. Cell Tissue Res 247:59-66.

Ribi WA. 1974. Neurons in the 1st synaptic region of the bee Apis-mellifera. Cell Tissue Res 148:277-286.

Ribi WA, Scheel M. 1981. The 2nd and 3rd optic ganglia of the worker bee Apis-mellifera Golgi studies of the neuronal elements in the medulla and lobula. Cell Tissue Res 221: $17-43$.

Rybak J, Menzel R. 1993. Anatomy of the mushroom bodies in the honey-bee brain - the neuronal connections of the alpha-lobe. I Comp Neurol 334:444-465.

Sachse S, Galizia CG. 2002. Role of inhibition for temporal and spatial odor representation in olfactory output neurons: a calcium imaging study. I Neurophysiol 87:1106-1117.

Salio C, Lossi L, Ferrini F, Merighi A. 2006. Neuropeptides as synaptic transmitters. Cell Tissue Res 326:583-598.

Schäfer S, Bicker G. 1986. Distribution of gamma aminobutyric-acid-like immunoreactivity in the brain of the honeybee Apis-mellifera. J Comp Neurol 246:287-300.

Schäfer S, Rehder V. 1989. Dopamine-like immunoreactivity in the brain and suboesophageal ganglion of the honeybee. I Comp Neurol 280:43-58.

Schürmann FW, Erber J. 1990. FMRFamide-like immunoreactivity in the brain of the honeybee (Apis mellifera). A lightand electron microscopic study. Neuroscience 38:797807.

Schürmann FW, Klemm N. 1984. Serotonin-immunoreactive neurones in the brain of the honeybee. I Comp Neurol 225:570-580.

Sinakevitch I, Niwa M, Strausfeld NJ. 2005. Octopamine-like immunoreactivity in the honey bee and cockroach: comparable organization in the brain and subesophageal ganglion. ] Comp Neurol 488:233-254.

Skiebe P, Schneider H. 1994. Allatostatin peptides in the crab stomatogastric nervous system: inhibition of the pyloric motor pattern and distribution of allatostatin-like immunoreactivity. J Exp Biol 194:195-208.

Stay B, Tobe SS. 2007. The role of allatostatins in juvenile hormone synthesis in insects and crustaceans. Annu Rev Entomol 52:277-299.

Stay B, Chan KK, Woodhead AP. 1992. Allatostatin-immunoreactive neurons projecting to the corpora allata of adult Diploptera-punctata. Cell Tissue Res 270:15-23.

Stay B, Tobe SS, Bendena WG. 1994. Allatostatins - identification, primary structures, functions and distribution. Adv Insect Physiol 25:267-337.

Strausfeld NJ. 2002. Organization of the honey bee mushroom body: representation of the calyx within the vertical and gamma lobes. I Comp Neurol 450:4-33.

Strausfeld NJ, Homberg U, Kloppenburg P. 2000. Parallel organization in honey bee mushroom bodies by peptidergic Kenyon cells. J Comp Neurol 428:760-760.

Sun XJ, Fonta C, Masson C. 1993. Odor quality processing by bee antennal lobe interneurons. Chem Senses 18: 355-377.

Suzuki H. 1975. Antennal movements induced by odours and central projections of the antennal neurons in the honeybee. J Insect Physiol 2120:831-847.

Takeda K. 1961. Classical conditioned responses in the honey bee. J Insect Physiol 6:168-179.

Takeuchi H, Yasuda A, Yasuda-Kamatani Y, Kubo T, Nakajima T. 2003. Identification of a tachykinin-related neuropeptide from the honeybee brain using direct MALDI-TOF MS and its gene expression in worker, queen and drone heads. Insect Mol Biol 12:291-298.

Takeuchi $H$, Yasuda A, Yasuda-Kamatani $Y$, Sawata M, Matsuo Y, Kato A, Tsujimoto A, Nakajima T, Kubo T. 2004. Preprotachykinin gene expression in the brain of the honeybee Apis mellifera. Cell Tissue Res 316:281-293.

Utz S, Schachtner J. 2005. Development of A-type allatostatin immunoreactivity in antennal lobe neurons of the sphinx moth Manduca sexta. Cell Tissue Res 320:149-162.

Utz S, Hütteroth W, Wegener C, Kahnt J, Predel R, Schachtner J. 2007. Direct peptide profiling of lateral cell groups of the antennal lobes of Manduca sexta reveals specific composition and changes in neuropeptide expression during development. Dev Neurobiol 67:764-777.

Veelaert D, Schoofs L, Tobe SS, Yu CG, Vullings HGB, Couillaud F, DeLoof A. 1995a. Immunological evidence for an allatostatin-like neuropeptide in the central-nervous-system of Schistocerca-gregaria, Locusta-migratoria and Neobellieria-bullata. Cell Tissue Res 279:601-611.

Veelaert D, Tobe SS, Yu CG, Schoofs L, DeLoof A. 1995b. Allatostatic and allatotropic factors in the brain of the desert locust, Schistocerca-gregaria. Belg J Zool 125:243-249.

Veelaert D, Devreese B, Schoofs L, VanBeeumen J, VandenBroeck J, Tobe SS, DeLoof A. 1996. Isolation and characterization of eight myoinhibiting peptides from the desert locust, Schistocerca gregaria: new members of the cockroach allatostatin family. Mol Cell Endocrinol 122: 183-190.

Vitzthum H, Homberg U, Agricola H. 1996. Distribution of dipallatostatin Hike immunoreactivity in the brain of the locust Schistocerca gregaria with detailed analysis of immunostaining in the central complex. I Comp Neurol 369: 419-437.

Weinstock GM, Robinson GE, Gibbs RA, Worley KC, Evans JD, Maleszka R, Robertson HM, Weaver DB, Beye M, Bork P, Elsik CG, Hartfelder K, Hunt GJ, Zdobnov EM, Amdam GV, Bitondi MMG, Collins AM, Cristino AS, Lattorff HMG, Lobo $\mathrm{CH}$, Moritz RFA, Nunes FMF, Page RE, Simoes ZLP, Wheeler D, Carninci $P$, Fukuda $S$, Hayashizaki $Y$, Kai $C$, Kawai J, Sakazume N, Sasaki D, Tagami M, Albert S, Baggerman $G$, Beggs $K T$, Bloch $G$, Cazzamali $G$, Cohen $M$, Drapeau MD, Eisenhardt D, Emore C, Ewing MA, Fahrbach SE, Foret S, Grimmelikhuijzen CIP, Hauser F, Hummon AB, Huybrechts J, Jones AK, Kadowaki T, Kaplan N, Kucharski $R$, Leboulle $G$, Linial $M$, Littleton $J T$, Mercer $A R$, Richmond TA, Rodriguez-Zas SL, Rubin EB, Sattelle DB, Schlipalius D, Schoofs L, Shemesh Y, Sweedler JV, Velarde R, Verleyen $P$, Vierstraete $E$, Williamson MR, Ament SA, Brown SJ, Corona $M$, Dearden PK, Dunn WA, Elekonich MM, Fujiyuki $T$, Gattermeier I, Gempe T, Hasselmann M, Kage E, Kamikouchi A, Kubo T, Kunieda T, Lorenzen M, Milshina NV, Morioka $M$, Ohashi $K$, Overbeek $R$, Ross CA, Schioett $M$, Shippy T, Takeuchi $H$, Toth $A L$, Willis $J H$, Wilson MJ, Gordon KHJ, Letunic I, Hackett K, Peterson J, Felsenfeld A, Guyer M, Solignac M, Agarwala R, Cornuet JM, Monnerot M, Mougel F, Reese IT, Vautrin D, Gillespie J], Cannone J, Gutell RR, Johnston JS, Eisen MB, Iyer VN, lyer V, Kosarev $P$, Mackey AJ, Solovyev V, Souvorov A, Aronstein KA, Bilikova $K$, Chen $Y P$, Clark $A G$, Decanini LI, Gelbart WM, Hetru $C$, Hultmark D, Imler $J$, Jiang $H B$, Kanost $M$, Kimura $K$, Lazzaro BP, Lopez DL, Simuth J, Thompson GJ, Zou Z, De Jong $P$, Sodergren $E$, Csuros M, Milosavljevic A, Osoegawa K, Richards S, Shu CL, Duret L, Elhaik E, Graur D, Anzola $J M$, Campbell KS, Childs KL, Collinge D, Crosby MA, Dickens $C M$, Grametes $L S$, Grozinger $C M$, Jones $P L$, Jorda $M$, Ling $X$, Matthews BB, Miller J, Mizzen C, Peinado MA, Reid JG, Russo SM, Schroeder A, St Pierre SE, Wang $Y$, Zhou 
PL, liang HY, Kitts $P$, Ruef $B$, Venkatraman A, Zhang L, Aquino-Perez G, Whitfield CW, Behura SK, Berlocher SH, Sheppard WS, Smith DR, Suarez AV, Tsutsui ND, Wei XH, Havlak P, Li BS, Liu Y, Jolivet A, Lee S, Nazareth LV, Pu LL, Thorn $R$, Stolc $V$, Newman $T$, Samanta $M$, Tongprasit WA, Claudianos C, Berenbaum MR, Biswas S, de Graaf DC, Feyereisen R, Johnson RM, Oakeshott JG, Ranson H, Schuler MA, Muzny D, Chacko J, Davis C, Dinh H, Gill R, Hernandez J, Hines S, Hume J, Jackson L, Kovar C, Lewis L, Miner G, Morgan M, Nguyen N, Okwuonu G, Paul H, Santibanez J, Savery G, Svatek A, Villasana D, Wright R. 2006. Insights into social insects from the genome of the honeybee Apis mellifera. Nature 443:931-949.
Witthöft W. 1967. Absolute Anzahl und Verteilung der Zellen im Hirn der Honigbiene. Z Morph 108:160-180.

Woodhead AP, Stay B, Seidel SL, Khan MA, Tobe SS. 1989. Primary structure of 4 allatostatins - neuropeptide inhibitors of juvenile-hormone synthesis. Proc Natl Acad Sci U S A 86:5997-6001.

Yoon JG, Stay B. 1995. Immunocytochemical localization of Diploptera punctata allatostatin-like peptide in Drosophila melanogaster. J Comp Neurol 363:475-488.

Zilberstein Y, Fuchs E, Hershtik L, Ayali A. 2004. Neuromodulation for behavior in the locust frontal ganglion. J Comp Physiol A Neuroethol Sens Neural Behav Physiol 190: 301-309. 